\title{
Nach dem Helsinki Gipfel - trübe Aussichten für eine Verbesserung der russisch-amerikanischen Beziehungen
}

https://doi.org/10.1515/sirius-2018-4005

Kurzfassung: Am 18. Juli 2018 fand in Helsinki ein Gipfeltreffen zwischen Präsident Donald Trump und dem russischen Präsidenten Vladimir Putin statt. Die vorliegende Untersuchung geht der Frage nach, ob die Idee des amerikanischen Präsidenten, mit Hilfe von Charme und Überredungskünsten im Vieraugengespräch mit dem KremlChef einen Durchbruch oder zumindest eine merkliche Verbesserung im russisch-amerikanischen Verhältnis zu erzielen, von Erfolg gekrönt war. Sie kommt zum Ergebnis, dass das Treffen nicht nur zu keinem Neustart und dem Beginn einer möglichen Bereinigung der Beziehungen beigetragen hat, sondern eher zu ihrer Verschlechterung. Die Gründe dafür lägen einerseits im Fehlen eines kohärenten Ansatzes Washingtons in der Politik gegenüber Moskau und andererseits an der Intransigenz der russischen Regierung, die den amerikanischen Präsidenten eher als Instrument zur Einschränkung der Handlungsfähigkeit der USA ansieht, denn als kompetenten Verhandlungspartner, mit dem eine Lösung der angehäuften Probleme in einer für beide Seiten akzeptablen Weise erfolgen könnte.

Schlüsselbegriffe: Russland, USA, Putin, Trump, Gipfeldiplomatie

Abstract: On 18 July 2018, a summit meeting was held in Helsinki between US-president Donald Trump and Russian president Vladimir Putin. The article raises the question of whether the idea of President Trump was crowned by success that he could achieve in one-on-one talks a breakthrough or at least a noticeable improvement in Russian-American relations based on his self-ascribed charm and persuasive abilities. The analysis arrives at the conclusion that the meeting not only failed to contribute to a restart of relations, but rather led to their further deterioration. The reasons for this are, on the one hand, the

*Kontakt: Hannes Adomeit, Senior non-resident Fellow, Institut für Sicherheitspolitik an der Universität Kiel (ISPK), Berlin, Deutschland; E-Mail: hannes.adomeit@t-online.de lack of a coherent approach in Washington's Russia policy and, on the other hand, the intransigence of the Russian government, which regards the US president more as an instrument for restricting US action, than as a competent negotiating partner with whom to solve the plethora of accumulated problems in a mutually acceptable manner.

Keywords: Russia, USA, Putin, Trump, summit diplomacy

\section{Einleitung}

Amerikanisch-russische wie früher amerikanisch-sowjetische Gipfeltreffen hatten oft einen herausgehobenen Charakter. Besonders in der Endphase des Ost-West-Konflikts kam ihnen eine zentrale Bedeutung $\mathrm{zu}$, weil auf ihnen wichtige politische Weichenstellungen erfolgten, die weitreichende Konsequenzen hatten. Ein erstes Gipfeltreffen zwischen Präsident Donald Trump und dem russischen Präsidenten Vladimir Putin fand am 18. Juli 2018 in Helsinki statt. In der Vorphase hatte der US-Präsident angekündigt, dass ihm an einer grundlegenden Verbesserung der Beziehungen zu Russland gelegen sei und er darüber mit dem Kreml-Chef persönlich und ohne jegliche weitere Teilnehmer, seien es Minister oder Berater, verhandeln wolle. Insofern wich der Gipfel nicht nur in diesem Punkt von allen vorangegangenen Gipfelkonferenzen mit Moskau ab, sondern auch hinsichtlich mangelnder Vorbereitung und fehlender Abstimmung zwischen dem Präsidenten und den mit Außen- und Sicherheitspolitik verantwortlichen Ministerien. Der Ausgang des Treffens traf in Washington und in den westlichen Hauptstädten überwiegend auf ein kritisches Echo und die Skeptiker sahen sich in ihren Befürchtungen bestätigt, dass Präsident Trumps Idee, mit Hilfe von Charme und Überredungskünsten im Vieraugengespräch einen Durchbruch im russischamerikanischen Verhältnis zu erzielen, zu nichts führen würde. Auf russischer Seite wurde der Gipfel anfangs als voller Erfolg gefeiert, mittlerweile hat sich auch in Moskau Ernüchterung eingestellt. 
Die der Untersuchung zugrunde liegende Arbeitshypothese ist wie folgt: Das Treffen in Helsinki hat nicht nur zu keinem Neustart und dem Beginn einer möglichen Bereinigung der russisch-amerikanischen Beziehungen beigetragen, sondern eher zu ihrer Verschlechterung. Um diese These $\mathrm{zu}$ verifizieren oder $\mathrm{zu}$ falsifizieren, wird als erstes der von Präsident Trump schon auf dem Treffen mit dem nordkoreanischen Staatschef Kim Jong-un in Singapur verfolgte Ansatz der persönlichen Gipfeldiplomatie vorgestellt. Danach werden die innenpolitischen Differenzen über die Russland-Politik in den USA und die unterschiedlichen Auffassungen zwischen dem Präsidenten auf der einen Seite und dem Kongress sowie den beteiligten Fachministerien seiner eigenen Administration auf der anderen Seite als Hindernisse für einen kohärenten Ansatz in der Russland-Politik Washingtons aufgezeigt. Dem werden die russischen Vorstellungen von einer Neuregelung der amerikanisch-russischen Beziehungen gegenüber gestellt. Dies führt zur Auflistung und Analyse der wichtigsten Streitpunkte im bilateralen Verhältnis. Im Ergebnis gelangt die Untersuchung zu dem Schluss, dass der Gipfel keines der aufgezeigten Probleme einer Lösung näher gebracht und die Beziehungen zwischen Moskau und Washington eher verschlechtert als verbessert hat. Ursache ist zum einen die Unerfahrenheit des amerikanischen Präsidenten und die Aussichtslosigkeit seiner Gipfelmethode, zum anderen ist es die Intransigenz der russischen Regierung, die den amerikanischen Präsidenten eher als Instrument zur Einschränkung der Handlungsfähigkeit des amerikanischen politischen Systems ansieht, aber nicht als kompetenten Verhandlungspartner, mit dem eine Bereinigung der Differenzen in einer für beide Seiten akzeptablen Weise erfolgen könnte.

\section{Die Methode der Trumpschen Gipfeldiplomatie}

Das Treffen von US-Präsident Donald Trump mit dem Kreml-Chef Vladimir Putin hat den Begriff der Gipfelkonferenz praktisch auf den Kopf gestellt. Gipfelkonferenzen lassen sich normalerweise wie eine Pyramide vorstellen. Auf breiter Basis finden auf Experten- und Ministerialebene umfangreiche Vorbereitungen statt. Die wesentlichen Streitpunkte, die geklärt werden sollen, sind bekannt und münden in eine Agenda und vorformulierte Texte möglicher Abkommen. Verläuft der Gipfel positiv, werden die Vereinbarungen in einem Abschlusskommuniqué auf einer gemeinsamen Pressekonferenz bekanntgegeben.
Dort werden gegebenenfalls auch verbleibende Aufgaben skizziert und nachfolgende Schritte umrissen.

Nichts davon trifft auf das Treffen in Helsinki vom 16. Juli 2018 zu. Als Spitze der Pyramide hat Trump versucht, eine sich von oben nach unten verbreiternde Basis für spätere Verhandlungen von Sicherheitsberatern und Ministern zu schaffen. So weit bekannt ist, gab es nur ein einziges Vorbereitungstreffen mit der russischen Führung - die Gespräche von Trumps Sicherheitsberater John Bolton am 27. Juni in Moskau. Eine Agenda mit konkret zu besprechenden Themen wurde nicht publiziert. Und als Trump einen Tag vor dem Gipfel gefragt wurde, welches Ziel er denn auf dem Treffen mit Putin verfolge, antwortete er: „Das werde ich ihnen nach dem Treffen sagen.“" Auf früheren sowjetisch- oder russisch-amerikanischen Gipfelkonferenzen war es üblich, dass bei ,Vieraugengesprächen" neben dem Übersetzer auch der Sicherheitsberater oder ein anderer Vertrauter des Präsidenten teilnahm. Auch dies war in Helsinki nicht der Fall. Nicht zuletzt deswegen ist bis heute - vermutlich auch für die engsten Mitarbeiter des Präsidenten - unklar, was in dem Gespräch und in welcher Form behandelt wurde. Noch Ende Juni hatte Putins außenpolitischer Berater in Moskau verkündet, dass ein gemeinsames Abschlusskommuniqué herausgegeben würde. ${ }^{2}$ Dies geschah aber nicht.

Offensichtlich war Trumps Gedanke für das Treffen in Helsinki derselbe wie der für sein Treffen mit Kim Jongun, das am 12. Juni 2018 in Singapur stattfand. Kraft seines Charmes, seines Verhandlungsgeschicks und seiner „Kunst, Deals zu machen,“3 würde er ein gutes persönliches Verhältnis zu Putin herstellen und die Grundlage für eine nachhaltige Verbesserung der russisch-amerikanischen Beziehungen schaffen. Allerdings ist genau das Gegenteil eingetreten. Das Verhältnis zwischen Moskau und Washington hat sich noch einmal verschlechtert, weil vor allem in Washington wie in den westlichen Hauptstädten die Kritik an Trumps Gipfeldiplomatie ebenso überhandgenommen hat wie das Misstrauen in sein Verhältnis zum russischen Präsidenten Vladimir Putin. Dieses Misstrauen war schon vorher da und ist vor allem vor dem Hintergrund der Ermittlungen zur russischen Einflussnahme

1 „I'll let you know after the meeting.” Trump auf die Frage des CBS-Reporters Jeff Glor, CNN, 15.7.2018, http://transcripts.cnn.com/ TRANSCRIPTS/1807/15/ndaysun.02.html.

2 Jurij Ušakov: Donald Trump, Vladimir Putin to hold summit in Finland on 16 July, security concerns on agenda, First Post, 28.6.2018, https://www.firstpost.com/world/donald-trump-vladimirputin-to-hold-summit-in-finland-on-16-july-security-concerns-onagenda-4622141.html.

3 „The Art of the Deal“ lautete der Titel des 1987 von Random House unter Trumps Namen publizierte Buch. 
auf die US Wahlen im November 2016 gestiegen und wurde weiterhin durch Gerüchte gestärkt, wonach Putin belastendes und kompromittierendes Material über Trump in der Hand hätte.

Anlass für die Kritik an Trump war dessen Auftritt in der Pressekonferenz zum Abschluss des Gipfeltreffens. Trumps Verhalten gegenüber Putin wurde in den Vereinigten Staaten teilweise mit Entsetzen und Empörung zur Kenntnis genommen. Dies betraf nicht nur die von Trump verunglimpften „liberalen Medien“ und die Demokraten, sondern auch führende Mitglieder der Republikanischen Partei. Er hätte sich von Putin wie ein Schulbube behandeln lassen, habe sich ihm gegenüber „unterwürfig“ verhalten und sich demütigen lassen. ${ }^{4}$ Der republikanische Senator John McCain, vor seinem Tod Ende August 2018 Vorsitzender des Streitkräfteausschusses des Senats, bezeichnete Trumps Auftritt in Helsinki als einen „historischen Tiefpunkt“. In seinen Augen sei die Pressekonferenz der beiden Staatschefs „eine der schändlichsten Aufführungen eines amerikanischen Präsidenten seit Menschengedenken“ gewesen und der Gipfel „ein tragischer Fehler.“5 Paul Ryan, republikanischer Vorsitzender des US-Repräsentantenhauses, stellte - entgegen den von Trump auch auf dem Gipfel geäußerten Zweifeln fest: „Es ist keine Frage, dass Russland in unsere Wahl eingegriffen hat und weiterhin versucht, die Demokratie hier und weltweit zu untergraben. Der Präsident muss anerkennen, dass Russland nicht unser Verbündeter ist.“6 Der republikanische Kongressabgeordnete und frühere CIA-Mitarbeiter Will Hurd teilte auf Twitter mit: „Ich habe in meiner beruflichen Karriere viele Menschen gesehen, die vom russischen Geheimdienst manipuliert wurden, und ich hätte nie gedacht, dass der US-Präsident einer

4 So wurde in der amerikanischen Berichterstattung über den Gipfel ausgiebig darauf hingewiesen, dass Putin zu dem, „historischen“ Treffen mit dem amerikanischen Präsidenten erst nach einer fünfunddreißigminütigen Verspätung erschien. Allerdings befand sich Trump dabei in bester Gesellschaft. Zu den Leidtragenden von Verspätungen des Kreml-Chefs gehören die Queen des britischen Königreichs drei Tage vor dem Helsinki-Gipfel (14 min) sowie Papst Franziskus im Juni 2015 (50 min). Den absoluten Rekord bisher nimmt das verspätete Erscheinen Putins zu einem Krisengipfel mit Bundeskanzlerin Angela Merkel im Oktober 2014 ein (4 h, 15 min), gefolgt von seinen Treffen mit dem früheren ukrainischen Präsidenten Viktor Janukovič im Oktober 2012 (4 h) und der damaligen ukrainischen Premierministerin Julia Timošenko im November 2009 (3 h).

5 Pressemitteilung McCains,16.7.2018, https://www.mccain.senate.gov/public/index.cfm? $p=$ press-releases\&id=A99FDA26-673D4560-B4EA-5AEDF0685EC5.

6 Vgl. House speaker says Trump 'must appreciate' Russia is not an ally, Reuters, 16.7.2018, https://www.reuters.com/article/us-usa-russia-summit-ryan/house-speaker-says-trump-must-appreciate-russiais-not-an-ally-idUSKBN1K62BZ. derjenigen sein würde, die von routinierten KGB-lern über den Tisch gezogen würden. “7 Der frühere CIA-Chef John Brennan warf Trump sogar „Hochverrat“ vor. ${ }^{8}$

Diese und andere vernichtende Urteile über Trumps Auftreten auf dem Gipfel deuten darauf hin, dass sich die Entschlossenheit in beiden Häusern des Kongresses und in beiden dort vertretenen Parteien noch weiter verstärkt hat, die Russland-Politik des Präsidenten, wie immer diese auch motiviert sein mag, nicht nur kritisch zu hinterfragen, sondern ihm auch Zügel anzulegen. In Grunde genommen macht der Kongress seine eigene Russland-Politik. Dies hatte sich bereits Ende Juli letzten Jahres gezeigt, als er ein Gesetz annahm, das neue Sanktionen gegen Russland beinhaltete und dabei bestimmte, dass der Präsident Sanktionen gegen Russland anwenden muss (mandatory sanctions) und gleichzeitig seine Befugnis beschneidet, bestehende Sanktionen zu verändern oder aufzuheben. ${ }^{9}$ Das Gesetz wurde mit überwältigenden Mehrheiten angenommen - mit 98:2 Stimmen im Senat und 419:3 im Repräsentantenhaus. Trump blieb nichts Anderes übrig, als das Gesetz zu unterzeichnen, denn sein Veto wäre problemlos aufgehoben worden. Das Misstrauen gegen Trump schlug sich auch in der Forderung nieder, die amerikanische Übersetzerin auf dem Helsinki-Gipfel zu einem Hearing in den Auswärtigen Ausschuss des Senats zu laden, um über den Inhalt des Gesprächs zu berichten. ${ }^{10}$

Rund eine Woche nach dem Helsinki-Gipfel kündigten der republikanische Senator Lindsey Graham und Bob Menendez von der Demokratischen Partei an, dass sie einen neuen Gesetzentwurf zur Ausweitung der gegen Russland gerichteten Sanktionen im Senat einbringen würden. Gleichzeitig machten sich die Senatoren Marco Rubio von den Republikanern und Chris Van Hollen von den Demokraten für einen anderen Gesetzentwurf über neue Sanktionen stark, die dann verfügt werden könnten, wenn nicht der Präsident, sondern der Direktor für nationale Aufklärung bestätigen würde, dass sich Russland in die kommenden Wahlen (im November) einmische. Am 2. August 2018 brachten Graham und Menendez ihren Entwurf im Senat ein, der Beschränkungen für neue russische Staatsanleihen, Energie- und Ölprojekte und russische Uranimporte sowie neue Sanktionen gegen russische Politiker und Oligarchen vorsieht. Zudem würden

7 Hurd, 16.7.2018 @HurdOnTheHill.

8 John O. Brennan, 16.7.2018 @John Brennan.

9 U.S. Congress, Countering Russian Influence in Europe and Eurasia Act of 2017. 11th U.S. Congress, H.R. 3203, https://www.congress.gov/ bill/115th-congress/house-bill/3203/text.

10 Treibende Kraft hinter dieser Forderung war Senatorin Jeanne Shaheen von der Demokratischen Partei, ein Mitglied des Senate Foreign Relations Committee. 


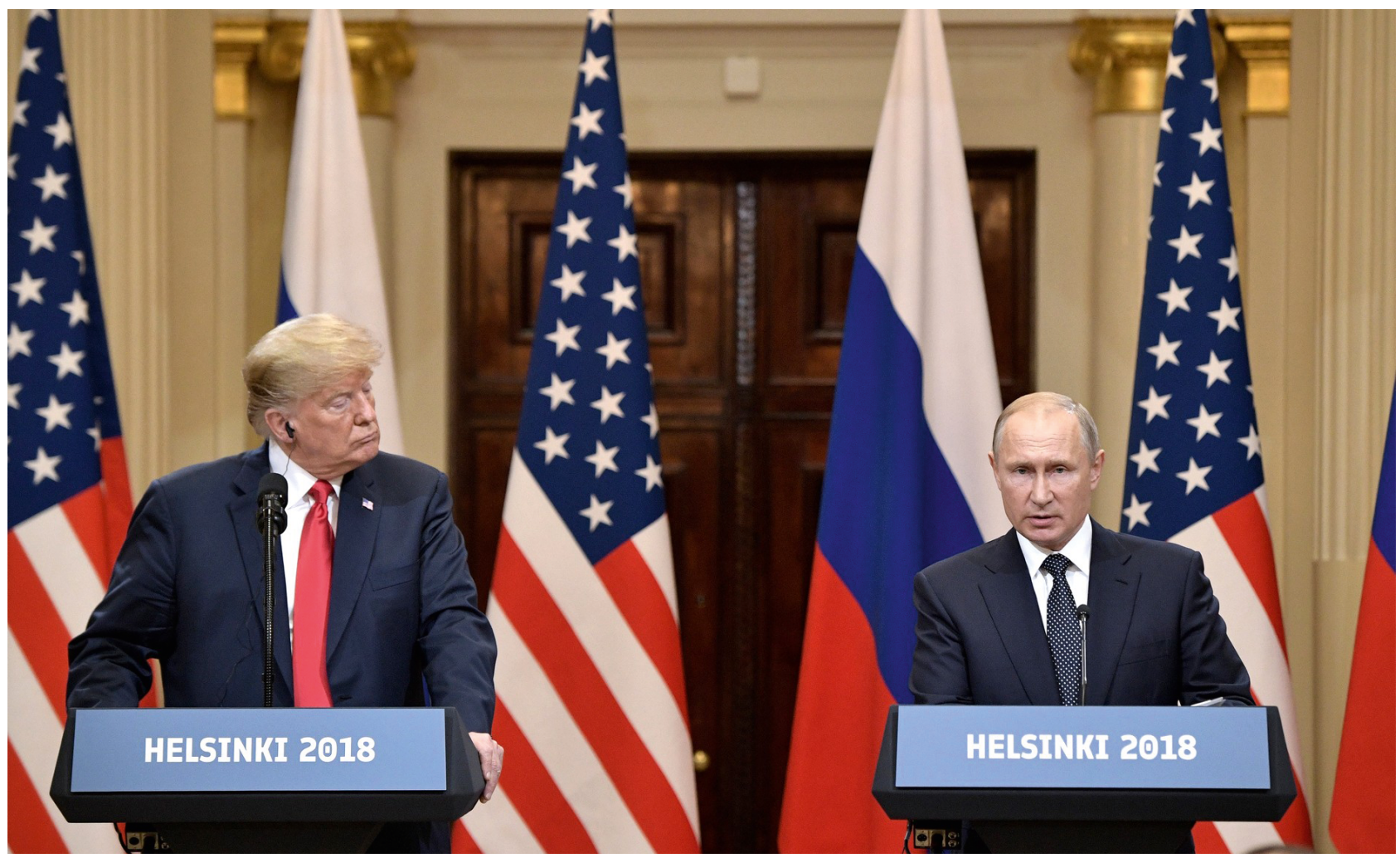

Abb. 1: Präsident Trump und Präsident Putin auf der Pressekonferenz am 16. Juli 2018 in Helsinki, Quelle: Creative Commons, Webseite des Russischen Präsidialamtes

sich erweiterte Strafmaßnahmen gegen die größten staatlich kontrollierten Banken Russlands richten und deren Dollar-Geschäfte einfrieren. Als Begründung gaben die Sponsoren des Gesetzentwurfs an, dass die bisherigen Sanktionen noch nicht ihre Wirkungen gezeigt hätten und der Präsident zudem zögere, diese auszuführen, es also an der Zeit sei, die Strafmaßnahmen drastisch zu verschärfen und „Sanktionen aus der Hölle“ zu verfügen. ${ }^{11}$

Es ist nicht nur der Kongress, der praktisch eine eigene, vom Präsidenten abweichende, schärfer gegenüber Russland akzentuierte Politik betreibt, sondern auch die mit Außenpolitik sowie innerer und äußerer Sicherheit befassten Ministerien und ihnen nachgeordneten Ämter der Exekutive. So gab der stellvertretende US-Justizminister Rod Rosenstein unbeeindruckt von den Zweifeln und Euphemismen Trumps drei Tage vor Helsinki bekannt, dass das Ministerium zwölf Mitarbeiter des russischen Militärgeheimdienstes GRU unter Anklage gestellt hätte. Ihnen würde vorgeworfen, für Hackerangriffe während des Wahlkampfs 2016 verantwortlich zu sein. Auf dem

11 Patricia Zengerle: U.S. senators introduce Russia sanctions 'bill from hell', Reuters, 2.8.2018, https://www.reuters.com/article/ususa-russia-sanctions/us-senators-introduce-russia-sanctions-billfrom-hell-idUSKBN1KN22Q.
Gipfel wiederholte Trump jedoch seine Zweifel an den Erkenntnissen seiner Geheimdienste und berichtete, dass er Putins Beteuerungen, Russland habe nichts mit den Cyber-Attacken zu tun, ,extrem stark und kraftvoll“ gefunden hätte und er (Trump) im Übrigen ,keinen Grund sehe, warum Russland so etwas tun sollte“. ${ }^{12}$ Diesen Relativierungen widersprachen die Sicherheitschefs vehement auf einer präzedenzlosen Pressekonferenz am 3. August 2018 in Washington. Die offensichtliche Einmischung Russlands mit Hilfe illegaler Wahlkampffinanzierung, Manipulation der sozialen Medien und Cyber-Angriffen auf die Wahlinfrastruktur 2016 sei keineswegs Geschichte, sondern die Anstrengungen Moskaus um Einflussnahme würden fortgesetzt. „Wir sehen weiterhin eine breit angelegte Kampagne Russlands, die Vereinigten Staaten zu schwächen und zu teilen“, erklärte Dan Coats, der Direktor für Nationale Aufklärung. ${ }^{13}$ Kirstjen Nielsen, die Minis-

12 "I don't see any reason why it would be [Russia] that interfered in the 2016 election", hatte er in Helsinki gesagt. Zwei Tage nach der Gipfelkonferenz bemühte er sich per Twitter, das Gegenteil zu erwecken. Er hätte gemeint: „I don’t see any reason why it wouldn't be [Russia].” (Hervorhebung nicht im Original.)

13 Rhys Blakely: US security chiefs: our country is in Russian crosshairs, The Times (London), 3.8.2018, 
terin für Heimatschutz, pflichtete ihm bei und meinte: „Unsere Demokratie ist im Fadenkreuz [Russlands]“..14 Auch für FBI-Direktor Christopher Wray war es offensichtlich, dass „sich Russland bis zum heutigen Tage bemühe, Operationen bösartiger Einflussnahme durchzuführen.“"15

Während der Vorbereitung des Graham-Menendez Gesetzes ergriff auch das Außenministerium die Initiative. Am 8. August 2018 teilte es mit, dass neue Strafmaßnahmen gegen Russland verhängt würden. Diese wurden mit dem Giftgasangriff auf den ehemaligen russischen Geheimdienstmitarbeiter Sergej Skripal und seine Tochter begründet. Die russische Regierung habe „chemische oder biologische Waffen unter Verstoß gegen internationales Recht eingesetzt oder tödliche chemische oder biologische Waffen gegen ihre eigenen Staatsbürger benutzt.“ Die ersten Sanktionen, die am 22. August 2018 in Kraft traten, verbieten Exportlizenzen an Russland für den Kauf von Gütern mit Bezug zur nationalen Sicherheit. Darunter fallen etwa Gasturbinen-Triebwerke und Elektronik- und Kalibriergeräte, die zuvor im Einzelfall exportiert werden durften. Vorher bestanden bereits Beschränkungen für den Export anderer militärischer und sicherheitsrelevanter Güter. Nach Angaben des US-Außenministeriums könnte ein Handelsvolumen von mehreren Hundert Millionen Dollar betroffen sein.

\section{Russische Kalkulationen und Reaktionen}

Putin und Mitglieder der russischen Delegation konnten ihre Begeisterung über den vermeintlichen Erfolg auf dem Helsinki-Gipfel vom 16. Juli 2018 kaum verbergen. Der Kreml-Chef strahlte vor Wohlgefallen und guter Laune auf der Pressekonferenz, die dem zweieinhalbstündigen Gespräch mit dem amerikanischen Präsidenten Donald Trump und dem sich daran anschließenden Mittagessen mit Mitgliedern der Delegationen folgte. Der sonst so reserviert und emotionslos auftretende russische Außenminister Sergej Lavrov war laut dem früheren amerikanischen Botschafter in Moskau, Michael McFaul, ganz aufgedreht und „trunken“ vor Freude. ${ }^{16}$ Reportern sagte er, dass

14 Ebd.

15 Ebd.

16 Vgl. Michael McFaul: The Trump-Putin summit in Helsinki was a historic event - in the worst possible way, Washington Post, 17. Juli 2018. Nach seinen eigenen Beobachtungen in Helsinki war "giddy“ der Ausdruck, den er zur Charakterisierung der Befindlichkeit Lavrovs gebrauchte. Ganz unter dem Eindruck der Ereignisse urteilte McFaul, dass das Treffen ein „fantastic win for Putin“ gewesen sei. das Gipfeltreffen „besser als super“ gewesen sei. ${ }^{17}$ Für Vjačeslav Nikonov, Enkel des sowjetischen Außenministers Vjačeslav Molotov und Leiter der Russische Welt Stiftung, war Helsinki ein Erfolg, nicht nur weil dies Lavrov und die beiden Präsidenten bestätigt hätten, sondern davon zeugten auch die „vielen praktischen [Ergebnisse], die zum Teil schon implementiert worden sind." Die seit Jahrzehnten ausgesetzten Rüstungskontrollverhandlungen könnten wieder aufgenommen werden und viele andere eingefrorene Kanäle der Kooperation auftauen. ${ }^{18}$ Auch russische Wirtschaftsvertreter schöpften neue Hoffnung. Der „Geist“ der Gipfelkonferenz hätte neue Bedingungen für eine Ausweitung der Zusammenarbeit zwischen den beiden Ländern geschaffen, meinte Andrei Kostin, Chef der von amerikanischen Sanktionen betroffenen staatlichen Außenhandelsbank VTB. „Wir haben den ersten Schritt gemacht und wir können jetzt erwarten, dass unsere Kontakte sich weiter entwickeln." ${ }^{19}$

Ein von Kreml-nahen Experten für internationale Politik besonders hervorgehobener Beweis für den Erfolg von Helsinki war die Reaktion der amerikanischen Öffentlichkeit, die aus „negativer Berichterstattung der russophobisch und gegen Trump eingestellten liberalen Medien“ in Amerika bestand. ${ }^{20}$ Auch für Ivan Danilov von RIA Novosti, einer der wichtigsten russischen Nachrichtenagenturen, war der Helsinki-Gipfel ein Erfolg und der Mühe wert, „und sei es nur, um die Reaktion westlicher Politiker, Experten und Medienpersönlichkeiten genießen zu können. Nach ihrer Panik und ihrem Hass zu urteilen, hat [Putin] einen großen Erfolg erzielt; [er] hat buchstäblich den amerikanischen Informationsraum in die Luft gejagt." ${ }^{21}$

Kernpunkt des Kalküls des Kreml-Chefs war es offensichtlich ein enges persönliches Verhältnis zu Trump herzustellen und auf ihn - seine Vorstellungen und seine Politik - einzuwirken. Eines der diesem Kalkül zugeordneten Ziele könnte es gewesen sein, ihn entweder als Hebel für eine - im Sinne Moskaus - „Normalisierung“ des russisch-amerikanischen Verhältnisses zu nutzen oder aber, falls dies nicht möglich sein würde, als Instrument

17 Putin-Trump talks were better than super, says Russian top diplomat, TASS, 16.8.2018, http://tass.com/politics/1013439.

18 Sondersendung des Ersten Kanals des staatlichen russischen Fernsehens zur Gipfelkonferenz am 17.7.2018, https://www.youtube. com/watch?v=Fnx2naHiF1E.

19 Interview mit den staatlichen Fernsehsender Rossija 24, 18.7.2018, https://ria.ru/world/20180718/1524890293.html.

20 Nikonov, Sondersendung des Ersten Kanals des staatlichen russischen Fernsehens.

21 RIA Novosti, 17.8.2018, https://ria.ru/analytics/20180717/1524742 927.html. 
einzusetzen, um die Handlungsfähigkeit der amerikanischen Regierung zu untergraben und eine gegen Russland gerichtete Politikverschärfung zu verhindern. Putin war infolgedessen darauf bedacht, Trumps überbordendes Ego in Helsinki zu streicheln. Einmal nannte er den Republikaner sogar beim Vornamen (,Wir hatten ein gutes Gespräch. Ich hoffe, dass wir uns besser verstehen, und ich danke Donald dafür." ${ }^{22}$ Im Beisein von russischen Journalisten lobte Putin Trump als ,qualifiziert und gut vorbereitet“, der Amerikaner höre zu, lasse Argumente gelten, beharre aber auch auf eigenen Standpunkten. Wenn auch nicht unproblematisch für Trump und seine Zweifel an russischer Einmischung in die Wahlen 2016, so offenbarte Putin dennoch, wer sein Wunschkandidat war: „Ja, ich wollte, dass er [Trump] gewinnt, denn er redete über die Normalisierung der russisch-amerikanischen Beziehungen.“23

Im Wesentlichen kann man aber nicht von einem „Erfolg“ des Kremls in Helsinki sprechen. Der Gipfel hat zwar die Polarisierung und die chaotischen innenpolitischen Verhältnisse in den USA befördert und eine in sich geschlossene, einheitliche Russland-Politik Washingtons verhindert, letztlich sind aber die Beziehungen zu Moskau weiter vergiftet worden und der politische Handlungsspielraum Trumps, was immer auch seine Absichten in punkto Russland-Politik gewesen sein mögen, ist weiter eingeschränkt worden. Umgekehrt ist $\mathrm{zu}$ argumentieren, dass - wie so oft in der Geschichte der sowjetischen wie der russischen, post-sowjetischen Außenpolitik - Putin zwar ein Scharmützel, aber keine wichtige Schlacht, geschweige denn den Krieg gewonnen hat.

Allerdings war schon im Vorfeld des Gipfels erkennbar, dass der Kreml nicht davon ausging, dass mit Trump eine Generalbereinigung der Beziehungen erreicht werden könnte. Dies lässt sich den sieben von Putin in Helsinki gemachten Vorschlägen entnehmen.

1. Ein Expertenrat sollte geschaffen werden, der Politologen, prominente Diplomaten und ehemalige Militärexperten beider Länder einbezöge und der nach Möglichkeiten suchen würde, die russisch-amerikanischen politischen Beziehungen aus der Sackgasse zu führen.

22 Vladimir Putin: Meeting of ambassadors and permanent representatives of Russia, Kreml-Webseite, 19.7.2018, http://en.kremlin.ru/ events/president/news/58037.

23 Jeff Mason von der Reuters Nachrichtenagentur hatte gefragt: "President Putin, did you want President Trump to win the election and did you direct any of your officials to help him do that?" Sowohl die vom Weißen Haus als auch vom Kreml veröffentlichte Darstellung verwischt völlig die Klarheit der Aussage Putins; siehe Uri Friedman: Russian Speakers Explain What Putin Actually Said About Trump, The Atlantic, 18.7.2018, https://www.theatlantic.com/international/ archive/2018/07/putin-trump-election-translation/565481/.
2. Zudem sollte eine russisch-amerikanischen Arbeitsgruppe, bestehend aus hochrangigen Unternehmern und Geschäftsleuten, gegründet werden, die die Wirtschaftskooperation zwischen beiden Ländern voranbringen sollte.

3. Weiterhin sollte die bilaterale Anti-Terrorismus Arbeitsgruppe wieder ihre Arbeit aufnehmen, die früher auf der Ebene der stellvertretenden Außenminister getagt hatte.

4. Auch sollte eine Arbeitsgrupe über Cyber-Sicherheit eingerichtet werden, ein Gremium, das Putin bereits auf dem G20-Gipfel in Hamburg vorgeschlagen hatte.

5. Gespräche über militärische und sicherheitspolitische Fragen sollten geführt werden, einschließlich der Verlängerung des Vertrags über die Beschränkung der strategischen Offensivwaffen (New START), das amerikanische Raketenabwehrsystem, Probleme mit der Umsetzung des INF-Vertrags und das Verbot zur Stationierung von Waffen im Weltraum.

6. Washington und Moskau sollten sich zusammen um die Überwindung der humanitären Krise in Syrien bemühen und Voraussetzungen dafür schaffen, dass die Flüchtlinge in ihre Heimatorte zurückkehren könnten.

7. Die Ukraine betreffend sollte ein Referendum in den Separatistengebieten Luhansk und Doneck zur Bestimmung ihres Sonderstatus abgehalten werden.

Die ersten sechs dieser Vorschläge gab Putin auf der Pressekonferenz in Helsinki bekannt. Den siebten - Referendum in der Ostukraine - soll Putin laut russischem Botschafter in Washington im Vieraugengespräch mit Trump gemacht haben..$^{24}$ Wie der amerikanische Präsident darauf reagiert hat und ob, beziehungsweise welche anderen Vorschläge der Kreml-Chef gemacht hat und auch gegebenenfalls, welche Vereinbarungen getroffen wurden, ist nicht bekannt. ${ }^{25}$

Nach Lage der Dinge sind die Chancen, dass auch nur ein einziger dieser Vorschläge in absehbarer Zeit erfolgreich verwirklicht werden könnte, gleich Null. Dies scheint auch Putin klar zu sein. Vermutlich in Anbetracht der vernichtenden Reaktion in den USA auf den Auftritt

24 Siehe Russia says Putin, Trump discussed a possible referendum in Ukraine, Reuters, 20.7.2018, https://www.cnbc.com/2018/07/20/ russia-says-putin-trump-discussed-referendum-in-ukraine.html.

25 Dies trifft ihren eigenen Aussagen zufolge auch auf höchste Beamte im außen- und sicherheitspolitischen Apparat zu. So sagte beispielsweise Dan Coats, der Direktor für nationale Aufklärung: „Ich weiß nicht, was in diesem Meeting [Trump-Putin Vieraugengespräch] passiert ist. Ich denke, im Laufe der Zeit ... werden wir mehr erfahren." Financial Times, 20.7.2018. 
Trumps wich Putin schon drei Tage nach dem Gipfel und noch vor den neuen amerikanischen Sanktionen in einer Rede von einem vorbereitenden Text ab und stellte fest: „Wir sehen, dass es Kräfte in den Vereinigten Staaten gibt, die versuchen, die Ergebnisse des Helsinki-Gipfels herunterzuspielen und zu verleugnen." Diese Kräfte setzten „enge Gruppen- und Parteiinteressen vor nationale Interessen" und setzten sich aus einflussreichen und mächtigen Leuten zusammen. Dies müssen ,wir in unserer praktischen Arbeit gegenüber den USA berücksichtigen.“26

Am 9. August 2018 befasste sich der Nationale Sicherheitsrat unter Vorsitz Putins mit den vom State Department angekündigten Sanktionen. Unter Bezugnahme auf die Sitzung bezeichnete Kremlsprecher Dmitrij Peskov die Strafmaßnahmen als ,absolut unfreundlich“, „völlig inakzeptabel“ und „illegal“; sie befänden sich auch nicht im Einklang mit der in Helsinki festgestellten „konstruktiven“ Atmosphäre. ${ }^{27}$

Als ähnlich ernst stufte Regierungschef Dmitrij Medvedev die neuen Sanktionen gegen sein Land ein. Diese kämen der „Erklärung eines Wirtschaftskrieges“ gleich. Sollten sich die angekündigten Sanktionen der USA etwa gegen Banken oder die Verwendung bestimmter Devisen richten, käme dies einer solchen Erklärung gleich. „Auf einen solchen Krieg müssen wir mit ökonomischen, politischen und falls nötig auch anderen Methoden reagieren, “ warnte er. ${ }^{28}$

Putin hat, wie sich herausstellt, den strategischen Fehler gemacht, voll und ganz auf Trump zu setzen. Diese Tatsache hat praktische Auswirkungen auf die Chancen einer nachhaltigen Bereinigung der russisch-amerikanischen Beziehungen, soweit diese den vom Kreml definierten russischen Interessen dienen soll. Was aber sind die Bedingungen, unter denen Moskau zufolge die Verbesserung des Verhältnisses stattfinden soll? Welche Forderungen müssten von Washington erfüllt werden?

Die Rekonstruktion der Bedingungen des Kremls sollte an der traditionellen russischen Doppelfrage ansetzen: „Kto vinovat?“ (Wer ist schuld?) und „Što delat'?“ (Was ist zu tun?). Die Antwort des Kremls auf die erste

26 Vladimir Putin: Podpisan zakon o priostanovlenii dejstviya sogla eniya me жdu Rossiej i SŠA ob utilizacii plutoniya [Das Gesetz über die Ausführung des zwischen Russland und der USA geschlossenen Vertrags über die Nutzung von Plutonium wurde unterzeichnet], Kreml-Webseite, 31. Oktober 2016, http://kremlin.ru/acts/news/53167. (Hervorhebung nicht im Original).

27 Kreml äußert sich zu neuen US-Sanktionen, Sputnik Nachrichtenportal, 9.8.2018, https://de.sputniknews.com/politik/201808 09321887925-peskow-sanktionen-usa/.

28 Kommersant, 10.8.2018, https://www.kommersant.ru/doc/3708 947.
Frage ist vorbehaltlos, dass Russland schuldlos sei. Für ihn liegt die Verantwortung für den Absturz der russischamerikanischen Beziehungen eindeutig und ausschließlich beim „Westen“ unter Führung der USA. Nach dem Zusammenbruch der Sowjetunion sei Washington von einer anti-sowjetischen zu einer anti-russischen Politik übergegangen. Es habe die Schwäche Russlands ausgenutzt, um Russland klein zu halten und ,auf die Knie zu zwingen“. Wichtigstes Instrument sei dabei die NATO mit ihrer Osterweiterung als der gegen Russland gerichteten Speerspitze gewesen. ${ }^{29} \mathrm{Um}$ eine nachhaltige Verbesserung des Verhältnisses zu erreichen, müssten also die Vereinigten Staaten ihre Russland-Politik fundamental ändern.

Das Prekäre an diesem Narrativ ist die Tatsache, dass der amerikanische Präsident sie zumindest in seiner öffentlichen Darstellung in wesentlichen Aspekten teilt. Das zeigt unter anderem seine Antwort auf die ihm vom Korrespondenten der Reuters Nachrichtenagentur in Helsinki gestellten Frage, ob er denn „Russland überhaupt für irgendetwas verantwortlich“ mache. „Ja“, antwortete Trump, „das tue ich. Ich halte beide Länder für verantwortlich. Ich denke, dass die Vereinigten Staaten dumm waren. Ich denke, wir waren alle dumm. Wir hätten diesen Dialog schon vor langer Zeit haben sollen - eine lange Zeit, ehrlich gesagt, bevor ich ins Amt kam. “30 Während er damit noch grundsätzlich beiden Staaten die Schuld am Zerwürfnis gibt, siedelt er doch die Verantwortung für die gegenwärtige Krise bei den Vereinigten Staaten an: „Ich denke, dass die Sonderuntersuchung [des Ermittlers Robert Mueller] eine Katastrophe für unser Land ist. Ich denke, sie [die Sonderermittlung] hat uns [USA und Russland] getrennt. Sie hat uns getrennt gehalten. Es gab überhaupt keine Absprachen. Jeder weiß es. Aber die Hauptsache, und wir haben das auch besprochen, es gab keine Verschwörung.“" ${ }^{31}$ Diese Sicht entspricht der russischen Erzählung, dass Trump ein Russland wohlgesonnener Politiker sei, dem durch ,gewisse Kräfte“ im Washingtoner Establishment (Trump: „Washington swamp“) die Hände gebunden werden.

Was nun ist $\mathrm{zu}$ tun? Was sind die Bedingungen, die der Kreml gewährleistet sehen will, um einen Neustart nach seinen Vorstellungen zu erreichen? Dies lässt sich

29 Zur Zurückweisung dieses Narrativs siehe Adomeit 2018a, 2018b und 2018c.

30 Vgl. White House: Remarks by President Trump and President Putin of the Russian Federation in Joint Press Conference, 16.7.2018, https://www.whitehouse.gov/briefings-statements/remarks-president-trump-president-putin-russian-federation-joint-press-conference/.

31 Ebd. (Hervorhebung nicht im Original). 
aus zweifelsfrei wiederholt vorgebrachten offiziellen Erklärungen Moskaus entnehmen. Washington müsse demnach

- den Dialog mit Moskau „auf Augenhöhe“ führen, Russland als „gleichberechtigten Partner“ ansehen und „mit Respekt“ behandeln,

- Bemühungen aufgeben, Russland oder anderen Ländern des postsowjetischen Raums das Regierungssystem der USA und ihre Lebensweise aufzuzwingen,

- den postsowjetischen Raum als Moskaus Einflusssphäre und Gebiet lebenswichtiger Interessen Russlands anerkennen, ${ }^{32}$

- Maßnahmen unterlassen, die darauf abzielen das zwischen den beiden Mächten existierende militärische Gleichgewicht zugunsten der Vereinigten Staaten zu verändern,

- jede weitere NATO-Osterweiterung rechtsverbindlich ausschließen,

- Pläne aufgeben, wonach Komponenten des nationalen amerikanischen Raketenabwehrsystems in Europa zu stationieren sind,

- sämtliche der seit August 2000 aufgestockten US-amerikanischen Truppen inklusive der dazugehörigen Infrastruktur in NATO-Ländern zurücknehmen, ${ }^{33}$

- sämtliche antirussischen Gesetze aufheben, einschließlich des sogenannten Magnitskij-Gesetzes aus dem Jahr 2012 und des Gesetzes zur Unterstützung der Freiheit der Ukraine vom 2014, welches die Grundlage für alle Sanktionen gegen natürliche und juristische russische Personen darstellt. ${ }^{34}$

- Russland für alle Verluste entschädigen, die es durch die Sanktionen und die darauf beruhenden Gegenaktionen erlitten hat. ${ }^{35}$

Wenn diese Bedingungen als Maximalforderungen anzusehen sind, die in Verhandlungen zurückgenommen oder zumindest erheblich modifiziert werden könnten, stellen sie doch wichtige Richtpunkte dar, die der Kreml nicht

32 Diese Forderung erhebt der Kreml nicht explizit. Sie lässt sich aber aus der Politik ableiten und wird von regierungstreuen Politikern und Propagandisten unterstützt.

33 Vladimir Putin: Podpisan zakon o priostanovlenii dejstviya sogla eniya me жdu Rossiej i SŠA ob utilizacii plutoniya [Das Gesetz über die Ausführung des zwischen Russland und der USA geschlossenen Vertrags über die Nutzung von Plutonium wurde unterzeichnet], Kreml-Webseite, 31. Oktober 2016, http://kremlin.ru/acts/news/53167. Diese Forderung wurde als Bedingung für die Wiederaufnahme der Maßnahmen zur Umsetzung des Abkommens zur beiderseitigen Vernichtung von atomwaffenfähigem Plutonium gestellt. Das Abkommen wurde im Dezember 2000 unterzeichnet.

34 Ebd.

35 Ebd. einfach aufgeben wird. Allerdings ist fraglich, ob der Kreml eine grundlegende Verbesserung des Verhältnisses zu den USA überhaupt will, bzw. ob er diese für realistisch hält. Tatsächlich verfolgt Russland weiterhin gegenüber den USA eine Politik, die die Konfrontation sucht und nur dann zu einer Annäherung führen könnte, wenn sich Washington ganz der russischen Sichtweise anschließt. Zudem ist absehbar, dass die eigenwillige Politik Präsident Trumps und der innenpolitische Streit zwischen der Administration und dem Kongress (den nicht zuletzt Putins Politik der Einmischung befördert hat) einen Reset in den Beziehungen zwischen beiden Ländern unwahrscheinlich werden lassen

\section{Differenzen bleiben bestehen}

Tatsächlich ist eher ist eine Komplizierung der Beziehungen und eine Vermischung mit anderen Problembereichen wahrscheinlich. Dies lässt sich anhand der Betrachtung der wichtigsten Streitpunkte aufzeigen, die derzeit die russisch-amerikanischen Beziehungen prägen. Darunter befindet sich der Streit über die weiterhin bestehende Einwirkung Russlands auf die amerikanische Innenpolitik, insbesondere die Versuche Wahlen zu manipulieren. Aber auch die Regelung der militärischen Konkurrenz zwischen Russland und den USA durch Rüstungskontrolle ist hier zu erwähnen. Dazu gehört auch die Frage ob das gemeinsame Interesse an der Bekämpfung des islamistischen Terrorismus Moskau und Washington näher zusammenführt und ob Russland den USA helfen werden, den Iran einzudämmen und zurückzudrängen. Zudem gehört das Verhältnis zu China zu diesem Kanon, insbesondere die Frage ob die USA Russland dazu gewinnen wollen eine Allianz gegen China zu schmieden. Auch die Annexion der Krim durch Russland und die andauernde Besetzung der Ostukraine sowie das Thema North Stream II gehören zu den Kernpunkten der russisch-amerikanischen Kontroverse. Der Gipfel von Helsinki hat wenig zur Lösung dieser Streitfragen beigetragen.

\subsection{Russische Einwirkung auf die ameri- kanische Politik und Gesellschaft}

Wie von den amerikanischen Geheimdiensten nachdrücklich und glaubhaft behauptet, geht das Bemühen des Kremls trotz aller ernsten Warnungen weiter, Dissens in der amerikanischen Politik und Gesellschaft zu säen und die Demokratie zu untergraben. Das wirft die Frage auf, 
warum dies der Fall ist, warum Putin eigentlich das Risiko akzeptiert, den Kongress und die amerikanische Öffentlichkeit noch weiter gegen Russland aufzubringen. ${ }^{36}$ Die Antwort ist in der russischen Innenpolitik und im Machterhaltungstrieb des Kremls zu finden. Seit der Endphase des Tandem-Arrangements mit Medvedev als Präsident und des Beginns seiner dritten Amtszeit ist Putin von einer sozio-ökonomischen Modernisierung des Landes mit westlicher Hilfe auf national-patriotische Mobilisierung übergegangen. ${ }^{37}$ Diese Wende geht mit einer scharf anti-westlichen und vor allem anti-amerikanischen Ausrichtung einher. Es ist ein Schwert mit scharfer Klinge auf beiden Seiten. Mit der einen soll liberaler westlicher Einfluss auf die russische Politik und Gesellschaft ausgeschlossen und mit der anderen das Image des Westens und wiederum insbesondere der USA als Beispiel von Demokratie und Rechtstaat untergraben werden. Beide Zielsetzungen werden derzeit vom Standpunkt der russischen Machtelite erfolgreich umgesetzt. Es gibt für sie infolgedessen keinen Grund, diese Ausrichtung grundsätzlich infrage zu stellen. ${ }^{38}$ Abgesehen von US Präsidenten Trump wird in den USA die russische Einmischung in den Wahlkampf vom Kongress als fundamentales Hindernis für eine Verbesserung der politischen Beziehungen gesehen und jede Fortsetzung dieser Einmischung wird eine wie auch immer geartete politische Konsenslösung zwischen Washington und Moskau verhindern. Der Gipfel hat diese Problematik nicht lösen können. Im Gegenteil, das Auftreten Präsident Trumps in der gemeinsamen Pressekonferenz mit

36 Laut CNN-Umfragen vom Juni und August 2018 hatten nur 11\% der Amerikaner eine positive Meinung zu Russland, $72 \%$ hatten unvorteilhafte Ansichten. Das waren die schlechtesten Ergebnisse für Russland seit Beginn der Umfragen (zur Sowjetunion) 1989; CNN 17.7.2018, https://edition.cnn.com/2018/07/17/politics/trump-russiarelations-poll/index.html und 17.8.2018, https://edition.cnn.com/ videos/politics/2018/08/17/cnn-poll-trump-too-friendly-with-russiasot-wolf-vpx.cnn.

37 Siehe die eingehenden Untersuchungen bei Adomeit 2017a und 2017b.

38 Allerdings muss der Kreml seinem innenpolitischen Machtkalkül entsprechend die Klingen immer wieder schärfen. Wie eine Umfrage der unabhängigen Forschungsorganisation Levada zeigt, hat sich die Zahl der den USA freundlich gegenüber gesonnenen Russen in den zwei Monaten vor dem Helsinki-Gipfel verdoppelt. Zweiundvierzig Prozent der Befragten gaben an, dass sie gegenüber den USA eine „positive“ Einstellung hätten. Im Mai desselben Jahres waren es nur 20 Prozent. Die Umfrage ergab auch, dass der Prozentsatz der Befragten mit einer unvorteilhaften Ansicht über die USA im selben Zeitraum von 69 Prozent auf 40 Prozent gesunken ist. Das bedeutet, dass die Russen die USA zum ersten Mal seit mehreren Jahren mehr positiv als negativ ansehen. Siehe JRL Russialist, 2.8.2018, http://russialist. org/jrl-newswatch-russian-pollster-reports-sharp-rise-in-positiveviews-of-u-s-levadaru/. seinem russischen Amtskollegen hat alle Bedenken der Seriosität und der Professionalität des US Präsidenten nur bestärkt.

\subsection{Die militärische Konkurrenz}

In der Vergangenheit, sowohl in der Sowjetära als auch nach dem Zusammenbruch der Sowjetunion, waren russisch-amerikanische Rüstungskontrollverhandlungen und -abkommen ein Mittel, um die Beziehungen auch in anderen Bereichen zu verbessern. Einen entsprechenden Vorstoß machte Putin in Helsinki, als er Gespräche über die Verlängerung von New START, den INF-Vertrag, das amerikanische Raketenabwehrsystem und das Verbot zur Stationierung von Waffen im Weltraum vorschlug. Mit an Sicherheit grenzender Wahrscheinlichkeit werden Rüstungskontrollverhandlungen dieses Mal kein Instrument für einen umfassenden Neustart sein. Das liegt unter anderem an der von Putin hervorgehobenen Bedeutung militärischer Faktoren in der Innen- und Wirtschaftspolitik und militärischer Macht als Statussymbol und Mittel wirksamer Außenpolitik.

Putin bezeichnet den militärisch-industriellen Komplex als einen „Motor“ und eine „Lokomotive“ für die Modernisierung der Gesamtwirtschaft. Zusätzlich zum Aufbau des Feindbilds USA und NATO wird damit die umfassende Modernisierung sowohl der konventionellen als auch der nuklearstrategischen Streitkräfte legitimiert. Ihre Ausführung vollzieht sich im Rahmen des laufenden Staatlichen Rüstungsprogramms (GPV-2020) und seiner Anpassung vom Februar 2018 für den Zeitraum von 2018-2027. Dafür sind 20 Billionen Rubel (357 Milliarden US Dollar) bereitgestellt worden, wovon 19 Billionen Rubel für die Entwicklung, Beschaffung und Reparatur militärischer Ausrüstung ausgegeben und die restliche Billion Rubel für den Bau der entsprechenden Infrastruktur verwendet werden sollen. Der Schwerpunkt des Programms liegt auf der weiteren Modernisierung der Nuklearwaffen und hochpräziser Luftraum- sowie Boden-Boden Systeme. ${ }^{39}$ Abstriche am Programm sind nicht vorgesehen und auch nicht wahrscheinlich.

Militärische Faktoren sind nahtlos in die russische Außenpolitik eingebunden. So gibt es im Moskauer außen- und sicherheitspolitischen Establishment absolut kein Verständnis für die Sicht, dass es - egal in welchem Konflikt - „keine militärische Lösung“ geben könne. Das

39 Bruce Jones/Craig Caffrey: Putin signs new State Armaments Programme, Jane's 28.2.2018, https://www.janes.com/article/78235/ putin-signs-new-state-armaments-programme. 
strategische Denken Russlands geht vielmehr davon aus, dass in einem Konflikt erst einmal die militärischen Verhältnisse geregelt werden müssen, um sich dann daran zu machen, die politischen Verhältnisse zu verändern. Von der Umsetzung dieses Ansatzes, also des Bemühens, militärische Erfolge in politische Veränderungen zu überführen, zeugen unter anderem die „Stabilisierung“ der Verhältnisse in Tschetschenien, die Anerkennung Südossetiens und Abchasiens nach dem Krieg mit Georgien, die Annexion der Krim nach der Invasion der Halbinsel durch die ,grünen Männchen“ und die Gründung der Separatistenrepubliken Lugansk und Doneck mit Hilfe russischer Truppen.

Die große Bedeutung, die Putin militärischen Faktoren in der Innen- und Wirtschaftspolitik und militärischer Macht in der Außenpolitik zubilligt, entspricht amerikanischem Denken auch unter Trump. Anders als im Bereich der Einmischung in Wahlen ist Präsident Trump hier kein Hoffnungsträger für russische Politik. Dieser hat vielmehr stets die These vertreten, dass Obama die USA als Militärmacht heruntergewirtschaftet und das Land deswegen international an Einfluss verloren habe. Diese Vorstellung beinhaltet eine Absage an jeglichen Gedanken von Mächtegleichgewicht und Rüstungskontrolle. Bereits in dem von ihm autorisierten Wahlprogramm der Republikanischen Partei hieß es: „Um es deutlich zu sagen, die US-amerikanische Militärmacht muss wieder zur stärksten der Erde mit umfassender Überlegenheit über jedes anderes Land oder jede Gruppe von Ländern der Welt aufgebaut werden. ${ }^{\text {" }}{ }^{0}$ Wie bei anderen Wahlversprechen macht Trump bei diesem Punkt ernst. So unterzeichnete er am 13. August 2018 den Verteidigungshaushalt für 2019 mit der Rekordsumme von 716 Milliarden Dollar - angeblich die „bedeutendste Investition in das Militär in der modernen Geschichte“. Trump wies auch emphatisch auf seine Pläne hin, eine Weltraumarmee (Space Force) zu schaffen. Es sei nicht ausreichend, dass Amerika im Weltraum lediglich präsent sei. „Wir brauchen eine Dominanz Amerikas im Weltraum.“ ${ }^{41}$ Er will eine Weltraumarmee zum eigenständigen sechsten Arm des Militärs machen. Eigene Weltraumstreitkräfte würden den Vereinigten Staaten Über-

40 Bei der Vorstellung des Wahlprogramms 2016, www.donaldjtrump.com/content-center/entry/obama-clintons-failure-with-russia. Pikanterweise saß bei Trumps Rede Russlands Botschafter in Washington in der ersten Reihe der Zuhörer.

41 President Signs Fiscal 2019 Defense Authorization Act at Fort Drum Ceremony, Press Release US Department of Defense, 13.8.2018, https://dod.defense.gov/News/Article/Article/1601016/presidentsigns-fiscal-2019-defense-authorization-act-at-fort-drum-ceremony/ und Trump unterzeichnet Verteidigungsetat über 716 Milliarden Dollar, FAZ, 14.8.2018. legenheit über ihre Rivalen geben. Diese hätten bereits begonnen, „den Weltraum zu bewaffnen“. Trump warnte in diesem Zusammenhang speziell vor China. Russland, das ebenfalls über „Weltraumtruppen“ verfügt, nannte er nicht.

Diese Unterlassung unterstreicht dann doch wieder die unterschiedlichen Vorstellungen zwischen Trump und den mit innerer und äußerer Sicherheit befassten Ministerien und Ämtern sowie dem Kongress bis hin zum Streitkräfteausschuss des Senats. Das Gesetz über den Verteidigungshaushalt trägt den Namen seines Vorsitzenden, John McCains, ${ }^{42}$ der Putin in den vergangenen Jahren wiederholt als „Schläger“, „Mörder“ und „Killer“ bezeichnet hat und für den Russland der Hauptgegner Amerikas ist. Bei der Unterzeichnung des Gesetzes vermied es der Präsident aber, ihn überhaupt zu erwähnen. Trumps Haltung zu Russland steht auch im Widerspruch zur amerikanischen nationalen Verteidigungsstrategie, die - in dieser Reihenfolge - China und Russland als „revisionistische Mächte“ bezeichnet, von denen die „zentrale Herausforderung für den Wohlstand und Sicherheit der USA“ ausginge und mit denen sich das Land in „einer langfristigen strategischen Konkurrenz" befände. ${ }^{43}$

Zwar hat Putin in Helsinki vorgeschlagen, Gespräche über die Verlängerung von New START, das amerikanische Raketenabwehrsystem, den INF-Vertrags und das Verbot zur Stationierung von Waffen im Weltraum geführt werden. Keine dieser Anregungen, sollten sie von den Vereinigten Staaten aufgenommen werden, wäre in der Lage, eine bedeutsame Veränderung der strategischen Konkurrenz oder der politischen Beziehungen zu bewirken: New START setzt den von beiden Seiten betriebenen Modernisierung der Nuklearwaffen keine Grenzen; die USA werden sich nicht von Russland vorschreiben lassen, wie sie ihr nationales Raketenabwehrsystem oder entsprechende regionale Systeme konfigurieren wollen; den INF-Vertrag verletzt Russland, gibt dies aber nicht zu; und da das Verbot zur Stationierung von Waffen im Weltraum nicht umfassend ist, wird die von Trump geforderte Weltraumarmee wohl ebenfalls trotz russischer Kritik aufgestellt werden.

Die unterschiedlichen Vorstellungen Trumps und der mit innerer und äußerer Sicherheit befassten Ministerien und Ämter wie auch der nationalen Verteidigungsstrategie werden auch bei der Bewertung der militärischen Lage in Europa (insbesondere in Nordosteuropa) und der Bedeu-

42 John S. McCain National Defense Authorization Act for Fiscal Year 2019, https://www.congress.gov/bill/115th-congress/house-bill/5515/ text. 43 US National Defense Strategy 2018. 
tung der NATO offensichtlich. ${ }^{44}$ Trumps Auftritte vor und auf dem Gipfel waren gekennzeichnet durch sein lauwarmes Bekenntnis zur NATO und die scharfe Maßregelung von Bündnispartnern, die ihren finanziellen Verpflichtungen nicht nachkämen. Der Bündnispartner Deutschland wurde deutlich abgestraft, Kritik an Russland als dem eigentlichen Verursacher der Sicherheitsprobleme der NATO kam bei ihm nicht vor. Diese Haltung unterscheidet sich deutlich von der nationalen Verteidigungsstrategie seiner Administration. Ihr zufolge ist „ein starkes und freies Europa, das an die Prinzipien von Demokratie, nationale Souveränität und Verpflichtung zu Artikel 5 des Nord-Atlantikvertrages gebunden ist, lebenswichtig für unsere Sicherheit “ ${ }^{45}$ Das Dokument betrachtet zudem eine starke NATO als wichtiges Instrument, um der von Russland ausgehenden strategischen Herausforderung zu begegnen. Dazu rechnet sie Moskaus Anstrengungen, „die internationale Ordnung zu unterhöhlen“, „,die Welt im Einklang mit seinem autoritären Modell zu formen“, „die Nordatlantische Allianz zu zerstören“, „neu aufkommende Technologien zu nutzen, um demokratische Prozesse in Georgien, auf der Krim und in der Ostukraine zu diskreditieren und zu untergraben“ und „ein Vetorecht über Nationen an seiner Peripherie hinsichtlich ihrer innerstaatlichen, wirtschaftlichen und diplomatischen Entscheidungen zu erlangen“.

Derartige Unstimmigkeiten im Auftreten der USAdministration geben natürlich Anlass für Sorgen, dass der russische Präsident Putin versuchen könnte daraus Kapital zu schlagen. Im Vorfeld des Helsinki-Gipfels gab es Befürchtungen, Trump könne im Vieraugengespräch mit Putin ähnliche substantielle Zugeständnisse hinsichtlich NATO-Übungen an der Peripherie Russlands machen wie kurz zuvor gegenüber dem nordkoreanischen Diktator Kim Jong-un in Singapur. Zielscheibe hätte dabei die weitere Ausführung der von Obama nach Moskaus Annexion der Krim 2014 auf den Weg gebrachten „European Reassurance Initiative“ (ERI) sein können, die die „territoriale Integrität“ der 28-Nato-Staaten garantieren soll sowie die dritte Militärmanöver der Serie „Atlantic Resolve.“ Diese Befürchtungen erfüllten sich nicht, stattdessen erhöhte der US-Kongress mit der Unterschrift Trumps die Finanzmittel für die ERI von 800 Millionen US Dollar auf 4,7 Milliarden

Diese Entwicklungen lassen eines deutlich erkennen: Der amerikanische Präsident Donald Trump hat keine

44 Siehe ausführlicher den Beitrag von Rainer Meyer zum Felde in diesem Heft sowie Adomeit 2018a.

45 US National Defense Strategy 2018 (Hervorhebung nicht im Original). konsistenten Vorstellungen davon wie die Beziehungen zu Russland gestalten werden können und die Bandbreite dessen eng begrenzt ist, was er Putin an Konzessionen im Bereich militärischer Fragen und bei militärischen Aktivitäten an Russlands Grenzen machen kann

\subsection{Der „Kampf gegen den islamistischen Terrorismus“}

Ein Bereich, in dem Trump immer wieder Anknüpfungspunkte für eine enge Kooperation mit Russland sieht, ist die Bekämpfung des islamistischen Terrorismus, insbesondere des Islamischen Staates (IS). Allerdings ist auch hier absehbar, dass die Erwartungen Trumps nicht in Erfüllung gehen und vielmehr sein ungeschicktes Verhalten von Moskau ausgenutzt wird, um die USA als Ordnungsmacht aus der Region zu verdrängen. Schon zu Zeiten der Obama-Administration war erkennbar geworden, dass Russland und die USA bei der Bekämpfung des IS sehr unterschiedliche Ziele verfolgten. Während Washington im Rahmen einer internationalen Koalition die Bekämpfung des IS direkt anging, hatte die seit Herbst 2015 anhaltende Militärintervention Russlands primär das Ziel, das Assad-Regime zu stützen. ${ }^{46}$ Mittlerweile ist Assad durch die kombinierte Hilfe Moskaus und Teherans wieder einigermaßen fest im Sattel. Die vereinten militärischen Kräfte von Russland, Syrien und Teilen der iranischen Revolutionsgarde und der von Iran unterstützten Hisbollah-Miliz kontrollieren heute wieder große Teile Syriens, lediglich die kurdischen Gebiete und die Provinz Idlib befinden sich noch in der Hand von Rebellen. Die Rolle der USA, die sich erfolgreich um die Bekämpfung des IS bemüht hatte, ist in Syrien marginal geworden, dies lassen die zwischen Russland, Iran und der Türkei geführten Konsultationen erkennen. Washington ist im SyrienKonflikt zu einem Beobachter herabgestuft worden.

Trump hat die ohnehin schwachen Karten Washingtons noch weiter abgewertet, indem er zu erkennen gab, dass er die amerikanischen Truppen aus Syrien so schnell wie möglich abziehen will. Der IS sei „fast vollständig vernichtet“, ließ das Weiße Haus im April 2018 verlauten. ${ }^{47}$ Die militärische Operation, IS in Syrien zu schlagen, ginge „schnell ihrem Ende entgegen“. Und Trump kündigte an: „Wir werden schnell aus Syrien herausgehen, sehr schnell

46 Bino/Krause 2017.

47 Pressesprecherin Sarah Sanders laut Julie Hirschfeld Davis: Trump Drops Push for Immediate Withdrawal of Troops From Syria, New York Times, 4.4.2018. 
sogar. Sollen sich andere Leute jetzt darum kümmern." ${ }^{48}$ Genau das aber ist das Problem, denn zu diesen ,anderen Leuten" gehören die Mullahs in Teheran - ein wesentlicher Grund dafür, dass Verteidigungsminister James Mattis und Generalstabschef Joseph Dunford den US-Präsidenten davon überzeugen konnten, den Gedanken des Truppenabzugs vorerst fallen zu lassen. ${ }^{49}$

\subsection{Die iranische Herausforderung}

Nachdem dem IS eine zusammenhängende große territoriale Operationsbasis entzogen worden ist, ist Iran als „größter staatlichen Sponsor des Terrorismus“ zum Hauptgegner der USA im Nahen und Mittleren Osten aufgerückt. Der bis weit in der Republikanischen Partei geteilten Sicht entsprechend ist die Islamische Republik noch gefährlicher als der IS, weil sie nicht nur die Fähigkeit, sondern auch den Willen habe, Nuklearwaffen zu produzieren. Diese Gefahr sei durch das Abkommen mit Iran zur Begrenzung seiner Nuklearaktivitäten (Joint Comprehensive Plan of Action - JCPOA) auf zivile Zwecke keineswegs gebannt worden - laut Trump der „schlechteste Deal, der je verhandelt wurde“, der den „Weg zur Atombombe“ nicht blockiert, sondern erst "freigemacht“ habe. Das Abkommen zu „zerlegen“ hatte er deshalb als außenpolitische „Priorität Nummer eins“ bezeichnet. ${ }^{50}$ In konsequenter Ausführung dieser Absicht gab er am 8. Mai 2018 bekannt, dass die Vereinigten Staaten aus dem Abkommen aussteigen würden. Im Anschluss unterzeichnete er eine Anweisung, durch die sämtliche Wirtschaftssanktionen, welche die USA wegen des Vertrags ausgesetzt hatten, wieder in Kraft gesetzt werden. Zudem kündigte er neue Wirtschaftssanktionen „auf höchstem Niveau“ an, um Druck auf Iran zum Abschluss eines neuen Abkommens und Aufgabe seiner außenpolitischen Ambitionen auszuüben. Die Strafmaßnahmen würden auch Länder betreffen, die Wirtschaftsbeziehungen zu Teheran unterhalten (extraterritoriale oder sekundäre Sanktionen), also auch Moskau.

Dem Konsens im Weißen Haus und unter den USRepublikanern entsprechend, dass Iran die „größte Bedrohung “ für amerikanische Interessen im Nahen und Mittleren Osten darstelle, ${ }^{51}$ befassten sich die Gespräche, die

\section{Ebd.}

49 Siehe auch den Beitrag von Jonathan Spyer in diesem Heft. 50 Sarah Begley: Read Donald Trump's Speech to AIPAC, Time Magazine, 22.3.2016, http://time.com/4267058/donald-trump-aipacspeech-transcript/.

51 So hatte US-Außenminister Mike Pompeo bei einer Anhörung im US-Kongress am 27.6.2018 ausgesagt: „Aus amerikanischer Sicht scheint mir der Iran die größte Bedrohung für die Vereinigten Staaten
Trumps Sicherheitsberater John Bolton am 27. Juni 2018 in Moskau in Vorbereitung auf den Helsinki-Gipfel führte, mit diesem Problem. Nach seinem Treffen mit Putin berichtete er, dass das Verbleiben Assads an der Macht für die USA keine strategisch bedeutsame Frage (mehr) sei. Die militärische Präsenz Irans sei allerdings ein Thema, „über das die beiden Präsidenten ausführlich sprechen wollen. Es gibt Möglichkeiten, größere Verhandlungen zu führen, um die iranischen Streitkräfte aus Syrien und zurück in den Iran $z u$ bringen, was ein bedeutender Schritt vorwärts wäre." 52

Was immer Putin und Trump auf dem Gipfel in Helsinki bezüglich Iran besprochen haben, bleibt im Dunkel. Offenkundig hat es keinen „Deal“ gegeben. Dafür gibt es viele Gründe. Einer davon ist die Unterschätzung des Stellenwerts, den Iran im Kalkül des Kremls einnimmt. Wie Teheran bezeichnet Moskau sein Verhältnis zur Islamischen Republik als „strategische Partnerschaft“. Wie übertrieben diese Stilisierung auch sein mag, für Russland ist Iran ein wichtiger Partner, um amerikanischen Einfluss im Nahen und Mittleren Osten zu begrenzen. In den letzten Jahren haben beide Länder in Syrien militärisch eng zusammengearbeitet. Schon die von Russland im Oktober 2015 von Kriegsschiffen aus dem Kaspischen Meer auf Ziele in Homs abgefeuerten Marschflugkörper vom Typ Kalibr 3M-14 überflogen iranisches Territorium mit Zustimmung Teherans. Im August 2016 erlaubte Iran der russischen Luftwaffe, seine Luftwaffenbasis Hamadan im Norden des Landes für Einsätze in Syrien zu nutzen. Russland profitiert finanziell vom Bau von Atomkraftwerken in Iran und potentiell von den Anstrengungen der Islamischen Republik, ihre Streitkräfte von Grund auf zu modernisieren. Dazu gehört ihre Ausrüstung mit S-300 Raketenabwehrsystemen, deren Lieferung im Wert von rund 800 Millionen US-Dollar Moskau 2007 vertraglich zugesichert hatte, dessen Ausführung der Kreml aber wegen des Konflikts über das iranische Atomprogramm lange Zeit aussetzte. Auch aus diesem Grund hat Moskau Interesse an der weiteren Gültigkeit des internationalen Atomabkommens mit Iran und der Beendigung des Sanktionsregimes.

Allerdings hat die absehbare Ausdehnung der Kontrolle Assads über das gesamte Land die Frage aufgeworfen, wie man mit der starken Präsenz der iranischen Revolutionsgarden und der Hisbollah und dem großen

zu sein und der Ort, auf den wir unsere Bemühungen konzentrieren sollten [...].“ Ebd.

52 Michael R. Gordon: Bolton Says Removing Iran From Syria Trumps Deposing Assad. National-security adviser says Trump wants Russia's assistance in getting Tehran's military forces to leave, Wall Street Journal, 1.7.2018 (Hervorhebung nicht im Original). 
Einfluss Teherans in Damaskus umzugehen soll. Putin hat dazu am 17. Mai 2018 im Rahmen eines Treffens mit Assad Stellung bezogen: „Wir gehen davon aus, dass angesichts der bedeutenden Siege und Erfolge der syrischen Armee im Kampf gegen den Terrorismus und dem Beginn einer aktiveren Phase des politischen Prozesses ausländische Streitkräfte aus dem Gebiet der Syrischen Arabischen Republik abziehen." ${ }^{53}$ Diese Positionierung bestätigte Außenminister Lavrov zwei Wochen später mit Blick auf die südwestliche Deeskalationszone (Provinzen Daraa, Quneitra und Suwayda): Alle „nicht-syrischen Kräfte“ müssten dieses Gebiet verlassen und das „so schnell wie möglich“. ${ }^{54}$ Und als Israel am 10. Mai 2018 in heftigen Angriffswellen iranische Militärziele in Syrien - praktisch die gesamte iranische Infrastruktur in Syrien - beschoss, enthielt sich Moskau jeglicher Kritik.

Auch wenn Putin im Rahmen einer politischen Nachkriegsregelung iranisches Militär und die Hisbollah aus Syrien heraus haben wollte, ist keineswegs klar, ob er auf die Mullahs entsprechenden Druck ausüben würde oder kann. Selbst wenn der Kreml bereit wäre, auf das Ansinnen Washingtons einzugehen, weiß er, dass seine Erfolgschancen unsicher wären. Die Islamische Republik ist kein von Moskau abhängiger Satellit, sondern verfolgt ihre eigenen Interessen. Diese bestehen unter anderem darin, eine zusammenhängende schiitische Landbrücke von Iran über den Irak und Syrien in den Südlibanon zu errichten. Außenminister Lavrov hat seiner Skepsis, erfolgreich auf Iran einwirken zu können, mit den Worten Ausdruck verliehen, das Land sei ein wichtiger Akteur im Nahen und Mittleren Osten und es sei „absolut unrealistisch“ zu erwarten, dass es sich aus Syrien zurückziehen und seine Interessen aufgeben würde. ${ }^{55}$ Somit ist eine Kooperation zwischen Washington und Moskau bezüglich der Einhegung und Eindämmung des iranischen Einflusses in der Region reichlich unwahrscheinlich. Der einzig existierende Kooperationsansatz - das JCPOA - wurde von der Trump-Administration versenkt.

53 Statements following Russian-Syrian talks, Kreml-Webseite, 17.5.2018, http://en.kremlin.ru/events/president/news/57488.

54 Lavrov: Pullback of non-Syrian forces from border with Israel must be fast, TASS, 30.5.2018, http://tass.com/politics/1007169. Die Deeskalationszone wurde von Russland, Jordanien und den Vereinigten Staaten im Juli 2017 vereinbart.

55 Russia: Complete Iranian withdrawal from Syria is 'absolutely unrealistic', Times of Israel, 4.7.2018, https://www.timesofisrael. com/russia-complete-iranian-withdrawal-from-syria-is-absolutelyunrealistic/.

\subsection{Die russisch-chinesische „strategische Partnerschaft" und die Denuklearisie- rung Nordkoreas}

Eine der möglichen Interpretationen für die von Trump an den Tag gelegte Putin- und Russlandfreundlichkeit könnte in der Hoffnung liegen, Russland zu einem strategischen Partner der USA im Konflikt mit China zu machen. Er scheint aus mehreren Gesprächen mit Henry Kissinger dessen Vorstellung übernommen zu haben, dass die langfristigen Interessen der USA und Russlands es erforderlich machten, „die gegenwärtigen Turbulenzen in ein neues Gleichgewicht zu verwandeln“. Russland sollte als „wesentliches Element eines neuen globalen Gleichgewichts wahrgenommen werden, nicht in erster Linie als Bedrohung für die Vereinigten Staaten." Putin, so Kissinger weiter, habe sich „übernommen, aber der Westen kann nicht dasselbe tun, oder er wird Russland in die Arme Chinas drängen und Machtvakua in Europa und Asien schaffen. ${ }^{656}$ Nicht nur in der amerikanischen Verteidigungsstrategie, wo China und Russland immer in dieser Reihenfolge genannt werden, sondern auch unter konservativen Vordenkern wird China langfristig als der größere Gegner der beiden Länder betrachtet. Trumps ehemaliger Sicherheitsberater Stephen Bannon hat einen Krieg mit China als „unvermeidlich“ bezeichnet und kürzlich sogar behauptet, dass „wir uns im Krieg mit China befinden“ und „wir gewinnen“. ${ }^{57}$ Dahinter steht der Gedanke, dass die USA sich mit Russland verbünden sollte, um China $\mathrm{zu}$ isolieren und zu schwächen, und dass Washington im Gegenzug dafür Moskau freie Hand in Ostmitteleuropa geben könnte.

Auch derartige Überlegungen sind auf Sand gebaut. Nach der Annexion der Krim, dem Ausbruch des Konflikts in der Ukraine und der Verhängung westlicher Sanktionen gegen Russland im Jahr 2014 verkündete Moskau lautstark eine „Wende nach Osten“, eine Neuausrichtung seiner Außen- und Wirtschaftspolitik weg vom Westen. Dem Kreml nahestehende Theoretiker wie Vladislav Surkov haben das Jahr 2014 als „Vollendung von Russlands epischer Reise in den Westen“ bezeichnet, als „das Ende wiederholter und fruchtloser Bemühungen, ein Teil der westlichen Zivilisation zu werden, um mit der ,guten Familie‘ der europäischen Völker eine Verbindung

56 Nahal Toosi: Kissinger, a longtime Putin confidant, sidles up to Trump, Politico, 24. Dezember 2016, http://www.politico.com/ story/2016/12/trump-kissinger-russia-putin-232925..

57 Bannon auf einer vom Wirtschafts- und Nachrichtensender CNBC organisierten Konferenz, CNBC, 18.7.2018, https://www.cnbc. com/2018/07/18/former-trump-advisor-steve-bannon-were-at-warwith-china.html. 
einzugehen““.$^{58}$ Derartigen Anschauungen zufolge soll eine engere Partnerschaft mit China eine lebensfähige Alternative zum Westen bieten. Entsprechend porträtiert der Kreml China (wie Iran) als „strategischen Partner“. ${ }^{9}$

So nimmt China schon seit Jahren an umfangreichen gemeinsamen Landmanövern mit Russland teil, unter anderem im September 2017 am russisch-weißrussischen Zapad- (Westen). Im September des 2018 Jahres waren chinesische Soldaten am Vostok- (Osten)-Großmanöver beteiligt; an der ersten Übung nahmen schätzungsweise bis zu 100.000 russische Soldaten teil, an der zweiten, dem größten Militärmanöver seit dem Ende des Kalten Krieges, sogar rund 300.000 Soldaten. Als solle die Symbolik dieser Partnerschaft auch im Syrien-Konflikt unterstrichen werden, hielten die beiden Länder im Mai 2015 Seemanöver im Mittelmeer ab. Im September 2016 nahmen russländische Kriegsschiffe mit der chinesischen Marine an Übungen im Südchinesischen Meer teil - offensichtlich eine demonstrative Unterstützung Moskaus für die territorialen Ansprüche Pekings in dieser Region. Im Juli 2017 schließlich setzten die beiden Länder mit gemeinsamen Seemanövern in der Ostsee ihre Demonstration gemeinsamer Übungstätigkeit und symbolhaft gemeinsamer strategischer Interessen fort. ${ }^{60}$

Wie das am 30. August 2018 abrupt aufgegebene Vorhaben Außenminister Pompeos zeigt, weitere Gespräche in Pjöngjang zu führen, war der Gipfelkonferenz-Ansatz Trumps der auf den Kopf gestellten Pyramide auch im nordkoreanischen Fall kontraproduktiv. Bis zum Treffen Trumps mit Kim Jong-un in Singapur schien es so, als wäre es Washington gelungen, mit Russland und China eine gemeinsame Drohkulisse gegen Pyöngjang aufzubauen. Sowohl für Putin und Xi Jinpin entfiel allerdings die Notwendigkeit des Drucks, als Trump gleich nach dem Gipfel verkündete: „Es gibt jetzt keine nukleare Bedrohung Nordkoreas mehr."61 Russland, so scheint es, ist (wie im iranischen Fall) nicht sonderlich daran interessiert, sich vorbehaltlos am Sanktionsregime gegen das Land $\mathrm{zu}$ beteiligen. Zumindest hat das US-Finanzministerium am 3. August 2018 neue Strafmaßnahmen verfügt, die auf eine russische Bank (Agrosojuz), zwei nordkoreanische Unternehmen und einen nordkoreanischen Bürger abzielen, um „illegale finanzielle Aktivitäten“ zu unterbinden. Am darauf folgenden Tag verhängte es weitere

58 Vladislav Surkov: Odinočestvo polukrovki [Die Einsamkeit eines Halbbluts], April 2018, http://www.globalaffairs.ru/number/-19490.. 59 Russisches Außenministerium [ohne Datum], http://www.mid. $\mathrm{ru} /$ strategiceskoe-partnerstvo-s-kitaem.

60 Bruns/Kirchberger 2017.

61 "There is no longer a Nuclear Threat from North Korea", Donald Trump @realDonaldTrump, 13.6.2018.
Strafmaßnahmen gegen Russen und mit Russland in Verbindung stehende Unternehmen. Die neuen Sanktionen richten sich gegen zwei russische, in Vladivostok ansässige Schifffahrtsunternehmen, Primorye Maritime Logistics und Gudzon Shipping. Die beiden Firmen sollen Erdölprodukte an nordkoreanische Schiffe weitergegeben haben, was laut Resolutionen des UN-Sicherheitsrates verboten ist. Außerdem strömen nordkoreanische Arbeiter weiter nach Russland, trotz UN-Sanktionen, die das Zurückfließen von Bargeld nach Nordkorea verhindern sollen.

Sollte Trump tatsächlich Putin anbieten, im Austausch gegen eine Verschärfung des Drucks auf Nordkorea und die „strategische Partnerschaft“ mit China zu verwässern, Konzessionen in Europa zu machen (zum Beispiel in der Krim-Frage und dem Konflikt in der Ostukraine), ist keineswegs sicher, dass er dies gegen den Widerstand des Kongresses und der mit Außenpolitik und Sicherheit befassten Ministerien und Ämter durchsetzen kann - und auch nicht, ob sich Russland darauf einlassen würde. Aber auch wenn es dies täte, wäre es früheren sowjetischen und post-sowjetischen - russischen - Verhaltensmustern durchaus entsprechend, wenn der Kreml die amerikanischen Konzessionen verbuchen, sein Verhalten gegenüber China aber nicht wesentlich verändern würde.

\subsection{Krim, Ostukraine und Sanktionen}

Wie bei nahezu allen anderen außen- und sicherheitspolitischen Fragen sind Haltung und Politik Trumps zur Krim und dem Konflikt in der Ostukraine sowie den damit verbundenen Sanktionen widersprüchlich und erratisch. Und auch bei diesem Themenkomplex offenbart sich eine tiefe Kluft zwischen Trumps Twitter-Diplomatie und den vom Kongress und der Regierung eingenommenen Positionen und getroffenen Maßnahmen.

Im Wahlkampf bis zu seinem Amtsantritt als Präsident erweckte Trump den Eindruck, als sei er an der Ukraine desinteressiert, könnte die Krim völkerrechtlich anerkennen und die gegen Russland gerichteten Sanktionen auch ohne substanzielle Gegenleistungen aufheben. Entsprechend hat Trump die strikte Bindung von Sanktionen an die vollständige Ausführung der Bestimmungen der Vereinbarungen von Minsk vom Februar 2015 relativiert. So sagte er beispielsweise eine Woche vor seinem Amtsantritt, er werde die Strafmaßnahmen „mindestens eine gewisse Zeit lang“ aufrechterhalten. Sollte man aber „miteinander auskommen“ und Russland „uns“ bei der Bekämpfung von Terroristen und der Erreichung anderer für die USA wichtigen Ziele „wirklich helfen“, dann könnten die Sank- 
tionen aufgehoben werden. ${ }^{62}$ Unbestritten ist außerdem, dass es vielfältige Kontakte von ehemaligen und heutigen Mitarbeitern Trumps zu russländischen Amtsträgern gab und es dabei auch um die mögliche Aufhebung von Sanktionen ging. ${ }^{63}$

Zudem löste Trumps Sicht der Dinge während des G7-Gipfels in Quebec im Juni 2018 große Besorgnis aus. Die Krim sei „ein Teil Russlands, weil jeder, der dort lebt, Russisch spricht”, sagte er auf dem Abendessen der G7. Auch wundere er sich, warum man sich auf die Seite der Ukraine gegen Russland stellen solle. Die Ukraine sei doch „eines der korruptesten Länder der Welt.“64 Zudem habe Russland für die Krim ,viel Geld ausgegeben, um es wieder aufzubauen“. Und schließlich überraschte er die Teilnehmer des Gipfels mit der Idee, dass Russland, das wegen der Krim-Annexion aus der G7 ausgeschlossen wurde, wieder in dieses Gremium zurückkehren solle. Trumps Ausführungen lösten eine Diskussion darüber aus, ob der amerikanische Präsident möglicherweise in Helsinki vorhätte, im Austausch gegen russische Zugeständnisse, die Halbinsel als russisches Staatsgebiet anzuerkennen. Die Diskussion über einen derartigen Deal wurde durch seine Bemerkungen auf der Pressekonferenz nach seinen Gesprächen mit Premierministerin Theresa May am 13. Juli 2018 beflügelt. Als er gefragt wurde, was denn seine Gedanken hinsichtlich einer Verbesserung der Beziehungen zu Russland seien, während Moskau doch „fortfahre, illegal ein anderes Land zu besetzen“, ging er in seiner Antwort weder auf die Völkerrechtswidrigkeit der Krim-Annexion noch auf die russische Intervention in der Ostukraine ein. ${ }^{65}$ Dafür gab er lediglich Präsident Obama die Schuld an dem „Desaster“ in der Ukraine.

Auch in seinem Eröffnungs-Statement auf dem Helsinki-Gipfel verlor Trump kein einziges Wort über die

62 Peter Nicholas/Paul Beckett/Gerald F. Seib: Trump Open to Shift on Russia Sanctions, 'One China' Policy. President-elect signals he would use any available leverage to realign U.S. relationship with its two biggest rivals, Wall Street Journal, 13.1.2017.

63 Beweise dafür sind die Gespräche Jared Kushners, Trumps Schwiegersohn, im Dezember 2016 mit Russlands Botschafter in Washington, Sergej Kisljak und mit Sergej Gorkov, dem Chef der staatlichen Vnešekonombank (VEB), einer Bank, die auf der US-amerikanischen Sanktionsliste steht. Ebenda.

64 So Trump während des G7-Abendessens laut einem Bericht von Buzzfeed, vgl. Alberto Nardelli/Julia Ioffe: Trump Told G7 Leaders That Crimea Is Russian Because Everyone Speaks Russian In Crimea, Buzzfeednews 14.6.2018, https://www.buzzfeednews.com/article/albertonardelli/trump-russia-crimea.

65 Briefing des Weißen Hauses, 13.7.2018, https://www.whitehouse. gov/briefings-statements/remarks-president-trump-prime-ministermay-united-kingdom-joint-press-conference/. konfliktträchtige Thematik. Putin erwähnte diese jedoch. „Während wir die innere Krise der Ukraine diskutierten“, berichtete er über das Vieraugengespräch, „haben wir auch der bona fide Implementierung der Vereinbarungen von Minsk durch Kiew besondere Beachtung geschenkt. Zugleich könnten die Vereinigten Staaten die ukrainische Führung entschlossener anregen und sie dazu bewegen, aktiver daran zu arbeiten." ${ }^{66}$ Mit anderen Worten, Putin wiederholte das offizielle russische Narrativ: Die internationalen Aspekte des Konflikts in der Ukraine hätten ihre Ursache in der anti-russischen Wende in diesem Land nach dem Maidan; dies erkläre den Stillstand bei der Ausführung der Minsker Vereinbarungen; Washington müsse infolgedessen Druck auf Kiew ausüben, um eine Lösung des Konflikts zu erreichen.

Nach den offiziellen Statements wurde Putin die Frage gestellt, ob Trump ihm eventuell etwas „hinsichtlich einer offiziellen Anerkennung der Krim als ein Teil Russlands“ gesagt hätte“. Putin berichtete, dass der amerikanische Präsident gemeint hätte, dass die Art und Weise, „wie die Krim Russland hinzugefügt wurde, illegal gewesen“ sei. ${ }^{67}$ Was Moskau anbeträfe, „haben wir ein Referendum in strikter Übereinstimmung mit dem Völkerrecht und der UN Charta durchgeführt.“ Der Fall sei „für Russland abgeschlossen." 68

Ob in dem Vieraugengespräch über die auch zwischen Washington und Moskau geführten Gespräche, eine UNFriedensmission im Donbass zu errichten, gesprochen wurde, ist nichts bekannt. Drei Tage nach dem Gipfel offenbarte Putin jedoch seinen Auslandsbotschaftern und Beamten des Außenministeriums in Moskau, dass doch über den Donbass gesprochen worden sei. Er hätte Trump den Vorschlag unterbreitet, ein Referendum unter internationaler Aufsicht in den Separatistenrepubliken Doneck und Luhansk über ihren Status durchzuführen. Es ist

66 White House 2018 (Hervorhebungen nicht im Original). Was Trump und Putin laut Transkript des Weißen Hauses (https://www. whitehouse.gov/briefings-statements/remarks-president-trumppresident-putin-russian-federation-joint-press-conference/) und des Kremls (http://en.kremlin.ru/events/president/news/58017) gesagt haben, unterscheidet sich zum Teil signifikant. So fehlt im vorliegenden Kontext in der Fassung des Kremls der Hinweis auf die ukrainische Innenpolitik, der eigentlich zum russischen Narrativ passt.

67 Der offiziellen russischen Sicht zufolge gab es keine Annexion der Krim. Entsprechend wird das, was Trump laut Putin gesagt habe, „richtiggestellt“. In der Version des Weißen Hauses heißt es: „He [Trump] continued to maintain that it was illegal to annex it." In der Kreml-Version liest man dagegen: „He [Trump] speaks about the illegal nature of annexing Crimea to Russia."

68 "This case is closed for Russia“ heißt es in der Kreml-Version. Im Transkript des Weißen Hauses ist zu lesen: „For us, this issue - we (inaudible) to this issue." 
zweifelhaft, ob Trump sich der Brisanz dieses Vorschlags bewusst war, denn sogenannte Referenda waren in den abtrünnigen Landesteilen der Ukraine ja schon im Mai 2014 durchgeführt worden. Damals sprachen sich angeblich 96 Prozent der abgegebenen Stimmen in den beiden „Volksrepubliken“ für ihre Unabhängigkeit aus. Einer der Zuhörer bei dem Treffen in Moskau berichtete, dass der amerikanische Präsident Putin zufolge darum gebeten hätte, den Referendumsvorschlag nicht zu erwähnen, weil er (Trump) Zeit benötige, um sich die Sache zu überlegen. ${ }^{69}$

Wie auch bei anderen wichtigen außen- und sicherheitspolitischen Fragen, scheinen der Präsident auf der einen Seite und die zuständigen Ministerien und Ämter sowie der US-Kongress auf der anderen auf völlig unterschiedlichen Schienen zu fahren. Dies deutete sich schon kurz nach Trumps Amtseinführung an. So warf die von Trump neu ernannte US-Botschafterin bei den Vereinten Nationen, Nikki Haley, Russland angesichts eskalierender Kämpfe in der Ukraine „aggressives Verhalten“ vor, das „klare und starke Verurteilung“ verdiene. Die USA werde „weiterhin die russische Okkupation der Krim verurteilen und zu deren unverzüglicher Beendigung auffordern.“ „Die Krim ist ein Teil der Ukraine“ und die Sanktionen würden infolgedessen „solange gelten, bis Russland die Kontrolle über die Halbinsel an die Ukraine zurückgegeben hat." ${ }^{\text {"70 }}$ Diese Äußerungen waren weit mehr als eine „Änderung der Tonart““ ${ }^{71}$ wie der russländische UN-Botschafter beklagte. Zur Bedingung für die Aufhebung der Sanktionen wurde nunmehr nicht nur die völlige Ausführung der Bestimmungen des Minsker Abkommens, sondern auch die Rückgabe der Krim erhoben. Haley fügte hinzu, dass der Präsident diese Forderung gegenüber Moskau „sehr klar gemacht“ habe. ${ }^{72}$ In Anbetracht von Trumps Verhalten in Helsinki ist allerdings zweifelhaft, ob dies tatsächlich der Fall war.

Die eklatanten Unterschiede zwischen Trumps Putinfreundlicher Haltung und Russland betreffenden Geset-

69 Ilya Arkhipov: Putin Lauds Trump's Summit Performance, Hits Out at U.S. Critics, Bloomberg News, 19. Juli 2018, https://www.bloomberg.com/news/articles/2018-07-19/putin-lauds-trump-s-summit-performance-hits-out-at-u-s-critics.

70 U.S. Ambassador to U.N. blames Russia for new violence in eastern Ukraine, Reuters, 2.2.2017, www.reuters.com/article/us-russiaun-ukraine-idUSKBN15H2TS?il=0.

71 Nicole Gaouette/Richard Roth: UN Ambassador Haley hits Russia hard on Ukraine, CNN News, 2.2.2017, https://edition.cnn. com/2017/02/02/politics/haley-russia-un/.

72 Trump expects Russia to return Crimea to Ukraine: White House, Reuters, 14.2.2017, https://www.reuters.com/article/us-usa-trumprussia-ukraine-idUSKBN15T2IY. zen und Maßnahmen der amerikanischen Regierung sind auch beim Problem der lange in den USA umstrittenen Lieferung von „tödlichen Waffen“ (lethal weapons) an die Ukraine klar erkennbar. Das betraf vor allem ,Javelin“ Panzerabwehrraketen. Im April 2018 wurden entsprechende Lieferungen begonnen. Die von Kritikern vorausgesagten militärischen Eskalationsrisiken haben sich nicht bewahrheitet. Zudem sollen die Waffenlieferungen ausgeweitet werden. Das hat der für die Verhandlungen mit Russland über eine UN-Blauhelmmission im Donbass zuständige US Regierungsbeauftragte Kurt Volker bestätigt. Die Ukraine, erläuterte er, „verliert jede Woche Soldaten, die ihr eigenes Land verteidigen. In diesem Zusammenhang ist es natürlich, dass sie ihr Militär aufbauen und ihre Verteidigung sicherstellen will.“ Ebenso natürlich sei es, „,dass sie Hilfe suchen will und andere Länder ihr helfen. Und natürlich brauchen sie Hilfe mit tödlichen Waffen, weil auf sie geschossen wird.“ In Anbetracht der Tatsache, dass die ukrainische Schwarzmeerflotte im Grunde genommen von Russland übernommen wurde, müsse die Ukraine „ihre Marine neu aufbauen [...] und wir müssen uns auch ihre Luftabwehr anschauen." ${ }^{\text {"73 }}$

\subsection{Nord Stream 2}

Die tiefe Kluft zwischen Trumps Putin-Apologetik und der per Gesetz vorgesehenen und von der US-Regierung ergriffenen Maßnahmen zeigt sich auch in einer sowohl zwischen Washington und Moskau als auch im transatlantischen Verhältnis umstrittenen Frage, dem Nord Stream 2 Projekt. In den Eingangsstatements auf der Pressekonferenz in Helsinki wurde das Projekt nicht erwähnt. Der Korrespondent der russischen Nachrichtenagentur Interfax stellte jedoch im Anschluss daran Trump folgende Frage: „Sie haben auf Ihrer letzten Europareise [in Brüssel am 11. Juli anlässlich Ihrer Teilnahme am NATOGipfel] erwähnt, dass die Umsetzung der Nord Stream 2-Gaspipeline Europa zur Geisel Russlands mache. Und Sie haben vorgeschlagen, dass Sie Europa davon befreien könnten, indem Sie amerikanisches Flüssiggas liefern.“ Das bestehende System der Gaslieferungen funktioniere aber gut. Die USA könne gar nicht die notwendigen Gasmengen nach Europa liefern. Wegen der geringen verfügbaren Angebotsmengen hätte die Stadt Boston sogar vor kurzem russisches Gas importieren müssen. Die Frage

73 Julian Borger: US ready to boost arms supplies to Ukraine naval and air forces, envoy says, Radio Free Europe/Radio Liberty, 1. September 2018, https://www.rferl.org/a/volker-says-us-ready-to-widenarms-supplies-to-ukraine/29465211.html. 
sei infolgedessen, ob Trumps Vorschlag wirklich „praktische“ Bedeutung habe, oder (lediglich) „politisch“ motiviert sei. ${ }^{74}$

In seiner Antwort ging Trump nicht auf die russische und ukrainische Dimension des Nord Stream 2-Projekts ein, sondern auf Deutschland. Er sei sich sicher, dass die USA erfolgreich mit LNG-Lieferungen konkurrieren können, obwohl es für Deutschland ,einen kleinen Standortvorteil“ gebe. Allerdings sei er sich „nicht sicher, ob die [Pipeline] im besten Interesse Deutschlands liegt oder nicht, aber das war eine Entscheidung, die Angela Merkel getroffen hat. [...] Also wünsche ich ihnen [den Deutschen] nur Glück.“ Dies kann man eigentlich nur so interpretieren, dass Trump zwar, wie er sagte, die Sache ,in ziemlich starker Tonart" mit der Kanzlerin besprochen habe, an der Verwirklichung der Gasleitung aber nicht mehr zu rütteln sei und er dies auch nicht vorhabe.

Bis zum 11. April 2018 war die Haltung der Bundesregierung eindeutig. Es leugnete konsequent den hochpolitischen Charakter von Nord Stream 2 und behauptete, es sei lediglich ein „kommerzielles Projekt“. Das Hauptproblem von Nord Stream 2 ist jedoch mit der russischen Politik gegenüber der Ukraine verbunden. Wenn die Pipeline gebaut und eine neue Pipeline in die Türkei das auf Eis gelegte South Stream-Projekt ersetzen würde, dann würde, wie Alexei Miller, CEO des russischen Energieriesen Gazprom, im Dezember 2014 in Ankara erklärte, die Rolle der Ukraine als Transitland „auf Null zurückgefahren“ ${ }^{75}$ Dies würde der Ukraine Einnahmen für die Durchleitung von russischem Gas nach Europa von jährlich 3 Mrd. USDollar entziehen, und es würde zu den Anstrengungen Moskaus passen, die Ukraine auch wirtschaftlich zu destabilisieren.

Bemerkungen Putins auf dem Helsinki-Gipfel zufolge hat Trump das Gasprojekt im Vier-Augengespräch angesprochen. „Der Präsident äußerte seine Besorgnis über Nord Stream 2 und die Möglichkeit des Verschwindens des Transitverkehrs durch die Ukraine“, berichtete er. „Und ich habe Herrn Präsident versichert, dass Russland bereit ist, diesen Transit aufrechtzuerhalten.“ Derartige Zusicherungen hatte Moskau schon in Gesprächen mit Minister Peter Altmaier in Moskau und mit der Bundeskanzlerin in Soči Mitte Mai 2018 gegeben. ${ }^{76}$ Die Frage ist

74 White House 2018.

75 Russia turns to Turkey in EU gas clash, Russia Beyond the Headlines, 16.12.2014, http://rbth.com/business/2014/12/16/russia_turns_ to_turkey_in_eu_gas_clash_42279.html.

76 Nachfolgende Darstellung laut: Altmaier hält Lösung für UkraineGas-Transit mit Russland für möglich, Handelsblatt 18.7.2018; Michael Bauchmüller: Altmaier im Minenfeld, Süddeutsche Zeitung, 14.5.2018; Russland will Gas weiter über Ukraine liefern, ZEIT Online, 18.5.2018, nur, zu welchen Bedingungen und mit welchem Volumen. In den Gesprächen mit den deutschen Regierungsvertretern hat Putin davon gesprochen, dass die Gaslieferungen über die Ukraine fortgesetzt werden könnten, solange diese „wirtschaftlich gerechtfertigt“ und „sinnvoll für alle Beteiligten“ seien. Gazprom-CEO Miller soll von der Bereitschaft seines Unternehmens gesprochen haben, 10-15 Milliarden Kubikmetern pro Jahr zu liefern. Solch ein Volumen würde aber kaum Rentabilitätskriterien erfüllen und ist weit entfernt von der in Berlin kursierenden Zahl von 38,5 Milliarden Kubikmeter für einen „substanziellen“ Fluss. Es gibt jedoch keine Anzeichen dafür, dass die russische Seite einer solchen Zahl zustimmen würde. ${ }^{77}$

Aber selbst unter der Annahme eines deutsch-russischen Abkommens über substanzielle Transitmengen könnte Nord Stream 2 noch verhindert oder erheblich verzögert werden. Dies könnte eine Folge der Ablehnung des Projekts durch die USA sein. Washingtons Gesetz zur Bekämpfung des russischen Einflusses auf Europa und Eurasien, das von überwältigenden Mehrheiten im Senat und im Repräsentantenhaus verabschiedet und am 2. August 2017 von Präsident Trump unterzeichnet wurde, ermächtigt die Regierung Sanktionen, einschließlich extraterritorialer Sanktionen, für eine Person oder Einrichtung vorzunehmen, die ,eine Investition [...] tätigt oder verkauft, oder Waren, Dienstleistungen, Technologie oder Informationen für den Bau russischer Energieausfuhrpipelines an Russland verpachtet oder bereitstellt“. Damit werden US-Sekundärsanktionen sowohl Gazprom als auch den an Nord Stream 2 beteiligten europäischen Unternehmen angedroht. Allerdings ist ihre Anwendung nicht zwingend vorgeschrieben (mandatory), sondern steht im Ermessen des Präsidenten (discretionary). Wie er sich entscheiden wird, ist auch in diesem Fall ungewiss. Die Position des Außenministeriums dagegen ist eindeutig. So sagte die stellvertretende US-Staatssekretärin Sandra Oudkirk, die für Energiefragen im State Department verantwortlich ist, bei ihrem einem Besuch am 17. Mai 2018 in Berlin, dass die Vereinigten Staaten gegen Nord Stream 2 seien, weil Energieprojekte dieser Art Russlands Potential zu „bösartigem Verhalten“ (malign behavior) erhöhten. Ehe Strafmaßnahmen tatsächlich angewandt würden, würde die USA aber erst alle diploma-

https://www.zeit.de/politik/ausland/2018-05/wladimir-putin-sotschi-gaslieferungen-ukraine-transitland; siehe auch Markus Decker: Die PD und die Russland-Frage, Berliner Zeitung vom 29.5.2018, S. 1.. 77 Diese Zahl errechnet sich durch Subtraktion der 55 Milliarden Kubikmeter der Nord Stream 2-Kapazität vom gegenwärtigen ukrainischen Transitvolumen. 
tischen Möglichkeiten ausschöpfen, um das Projekt zu verhindern, und würde dafür all ihre Überzeugungskraft einsetzen. ${ }^{78}$

\section{Fazit}

Das Treffen der Präsidenten Trump und Putin in Helsinki ist von beiden als großer Erfolg gefeiert worden. Der russische Außenminister fand es sogar „besser als super“. Tatsächlich hat der Gipfel die Polarisierung und chaotischen innenpolitischen Verhältnisse in den USA befördert und eine in sich geschlossene, einheitliche Russland-Politik Washingtons verhindert. Insoweit trifft die Sicht Moskaus zu, dass der Gipfel erfolgreich gewesen sei. Dies hat aber Washingtons Beziehungen zu Moskau weiter vergiftet und den Handlungsspielraum Trumps, was immer auch seine Absichten in punkto Russland-Politik sein mögen, weiter eingeschränkt. $\mathrm{Ob}$ dies richtig verstandenen russischen Interessen dient, ist zweifelhaft.

Eine zentrale Voraussetzung für eine nachhaltige Verbesserung der Beziehungen ist die Klärung der Frage, wer und was am Absturz der russisch-amerikanischen Beziehungen schuld ist. Die Verantwortung dafür schiebt der Kreml vorbehaltlos den USA zu. Die wahren Gründe liegen primär nicht in äußeren Herausforderungen oder „Bedrohungen“ Russlands wie beispielsweise der NATO-Osterweiterung, sondern sie sind in der russischen Innenpolitik, im Machterhaltungstrieb des Kremls, zu finden. Seit Beginn seiner dritten Amtszeit ist Putin von Medvedevs deklariertem Ziel einer sozio-ökonomischen Modernisierung des Landes mit westlicher Hilfe auf national-patriotische Mobilisierung übergegangen. Diese Wende geht mit einer scharf anti-westlichen und vor allem anti-amerikanischen Ausrichtung einher. Solange sich diese Politik nicht ändert und der Kreml meint, mit diesem Ansatz erfolgreich zu sein, können sich die russisch-amerikanischen Beziehungen und darüber hinaus das Verhältnis Moskaus zum Westen nicht ändern.

Die Aufgabe, die der amerikanische Präsident eigentlich hätte wahrnehmen müssen, um eine mögliche Richtungsänderung zu bewirken, wäre gewesen, das Narrativ des Kremls zurück zu weisen, auf die vielfältigen Beispiele für „bösartiges Verhalten“ des Kremls einzugehen und eine grundlegende Veränderung der russischen Politik anzumahnen. Die Wirksamkeit dieser Haltung

78 U.S. Warns Russian-German Gas Pipeline Risks Triggering Sanctions, Radio Free Europe/Radio Liberty, 18.5.2018, https://www.rferl. org/a/us-warns-russian-german-nord-stream-2-pipeline-risks-triggersanctions-security-concern-oudkirk/29233548.html. hätte dadurch erhöht werden können, wenn er dafür bei seiner Teilnahme an der NATO-Gipfelkonferenz in Brüssel und bei seinem Besuch in London starken europäischen Rückhalt gesucht hätte. Keinen dieser Schritte hat Trump unternommen. In Brüssel und London hat er die transatlantischen Beziehungen weiter abgewertet und in Helsinki beide Länder für die Zerrüttung des Verhältnisses verantwortlich gemacht, insbesondere aber, was das gegenwärtige Verhältnis anbeträfe, die amerikanische Innenpolitik in Form der Untersuchungen des Sonderermittlers Robert Mueller.

„No one has been tougher on Russia“ ist eine von Trump wiederholt aufgestellte Behauptung. Was ihn persönlich anbetrifft, ist diese Behauptung offensichtlich absurd. Das eigentlich unerklärliche Paradox seiner Haltung ist, dass er gegenüber Xi Jinpings China auf wirtschaftlicher und militärischer Stärke beruhenden Druck ausübt, gegenüber Putins Russland aber beschwichtigend auftritt - in Helsinki sogar laut seinen amerikanischen Kritikern „unterwürfig“. Scharfe Maßnahmen wie beispielsweise die nicht abreißen wollende Serie von Sanktionen sind ihm im Wesentlichen vom Kongress aufgezwungen oder von seinen Sicherheitsberatern und Ministern abgerungen worden.

Tatsächlich bewegen sich die gegenüber Russland vom US-Kongress und den mit innerer und äußerer Sicherheit befassten Ministerien und Ämtern in Washington eingenommene Haltung und verfolgte Politik einerseits und die des US-Präsidenten andererseits auf zwei fundamental unterschiedlichen Schienen. Erstere gehen in Übereinstimmung mit der amerikanischen Verteidigungsstrategie davon aus, dass Russland (wie China) eine „revisionistische“ Macht sei, die die auf liberalen und rechtstaatlichen Prinzipien beruhende internationale Ordnung grundlegend verändern wolle, und dass es Aufgabe amerikanischer Politik sein müsse, dieser großen strategischen Herausforderung zu begegnen und Russland Grenzen zu setzen. Trump scheint (trotz des sich abzeichnenden Fehlschlags im Verhältnis zu Kim Jong-un) weiter davon auszugehen, dass sein Charme und die Demonstrationen guten Willens verbunden mit wirtschaftlichen Anreizen die russisch-amerikanischen Beziehungen nachhaltig verbessern können. Dabei verkennt er das schon für die sowjetische Diplomatie von Stalin bis Brežnev gültige Handlungsprinzip von ,was mein ist, ist mein; was dein ist, ist verhandelbar". Überhaupt ist nicht offensichtlich, dass der Kreml eine grundlegende Verbesserung des Verhältnisses zu Washington will - es sei denn natürlich zu seinen Bedingungen. 


\section{Literatur}

Adomeit, Hannes (2013): Russisch-amerikanische Beziehungen: Fehler im Betriebssystem, in: Osteuropa, 63 (9) 57-78.

Adomeit, Hannes (2017a): Innenpolitische Determinanten der Putinschen Außenpolitik, Sirius: Zeitschrift für Strategische Studien, 1 (1), 33-52.

Adomeit, Hannes (2017b): Altes Denken, statt Neues Russland: Innenpolitische Bestimmungsfaktoren der russischen Außenpolitik, Portal Politikwissenschaft, 26.9.2017, https:// www.pw-portal.de/putins-russland/40508-altes-denken-stattneues-russland.

Adomeit, Hannes (2017c): Auf dem Tiefpunkt: USA-Russland Beziehungen unter Trump und Putin, in: Osteuropa, 67 (5), 115-133.

Adomeit, Hannes (2017d): Gas and Oil Interests Split Europe and US on Sanctions Policy, Raamoprusland (Niederlande), https:// www.raamoprusland.nl/dossiers/geopolitiek/636-gas-and-oilinterests-split-europe-and-us-in-sanction-policy.

Adomeit, Hannes (2018a): NATO-Osterweiterung: Gab es westliche Garantien? Berlin: Bundesakademie für Sicherheitspolitik, Arbeitspapier Sicherheitspolitik 3/2018.

Adomeit, Hannes (2018b): Russia's Alienation from the West: Who is to blame? Raamoprusland (Niederlande), https://www.raamoprusland.nl/debat/who-is-to-blame und https://www.integrityinitiative.net/articles/russiasalienation-west-who-blame.
Adomeit, Hannes (2018c): Will Trump and Putin bury NATO?, Raamoprusland (Niederlande), https://www.raamoprusland. $\mathrm{nl} /$ dossiers/geopolitiek/1014-will-trump-and-putin-burynato.

Bino, Tomisha/Krause, Joachim (2017): Russia Changes the Game - Bilanz der russischen Militärintervention in Syrien, Sirius - Zeitschrift für strategische Analysen, 1 (1), 68-79.

Bruns, Sebastian/Kirchberger, Sarah (2017): Machtprojektion ins NATO Meer. Die Marine der VR China in der Ostsee, Sirius Zeitschrift für strategische Analysen, 1 (4), 379-386.

Koerilla, Ivan (2018): Anti-Amerikanisme is spiegel van cultuurstrijd binnen Rusland, Raamoprusland (Niederlande), https://www. raamoprusland.nl/dossiers/geopolitiek/1037-betekenisen-oorsprong-van-het-anti-amerikanisme-in-rusland.

Krause, Joachim (2017): Die neue Zeitenwende in den internationalen Beziehungen: Konsequenzen für deutsche und europäische Politik, Sirius: Zeitschrift für strategische Studien, 1 (1), 3-24.

US Department of Defense (2018): National Defense Strategy, Washington, D.C., https://dod.defense.gov/Portals/1/ Documents/pubs/2018-National-Defense-StrategySummary.pdf 